Sharif University of Technology
Scientia Iranica
Transactions E: Industrial Engineering
http://scientiairanica.sharif.edu
IRAN ICA

\title{
Novel exponential divergence measure of complex intuitionistic fuzzy sets with an application to the decision-making process
}

\author{
H. Garg* and D. Rani \\ School of Mathematics, Thapar Institute of Engineering and Technology, Deemed University, Patiala 147004, Punjab, India.
}

Received 1 May 2019; received in revised form 9 September 2019; accepted 12 October 2019

\author{
KEYWORDS \\ Divergence measure; \\ Complex IFS; \\ Decision making; \\ Maximize deviation \\ method; \\ MCDM approach.
}

\begin{abstract}
As a generalization of Intuitionistic Fuzzy Sets (IFSs), Complex IFSs (CIFSs) are powerful and worthy tools for realizing the imprecise information using the complexvalued membership degrees with an extra term called phase term. Divergence measure is a valuable tool for determining the degree of discrimination between the two sets. Driven by these fundamental characteristics, it is fascinating to manifest some divergence measures under the CIFS theory. The present study proposed a method for solving the Multi-Criteria Decision-Making (MCDM) problem under CIFS environment. In this regard, firstly, the divergence measures were introduced between two CIFSs and their properties and relations were evaluated. Secondly, a novel algorithm was suggested based on the proposed measures to solve the problems in which the weights of the criteria were resolved by maximizing deviation method. Thirdly, a reasonable example was provided to verify the developed approach and exhibit its practicality and utility with a comparative analysis indicating its manageable and adaptable nature.
\end{abstract}

(C) 2021 Sharif University of Technology. All rights reserved.

\section{Introduction}

Nowadays, Decision-Making (DM) is one of the most significant ventures in our daily lives and its mission is to select the best alternative out of the finite options under several known or unknown criteria. MultiCriteria Decision-Making (MCDM) is the division of the DM regarded as a cognitive-based human action. Human beings inescapably face numerous DecisionMaking Problems (DMPs) which involve multiple fields such as supplier selection, supply chain management, emerging problems, medical problems, and so on. In practice, with the growing technological advancement

\footnotetext{
*. Corresponding author.

E-mail addresses: harishg58iitr@gmail.com (H. Garg); dimplegoyal938@gmail.com (D.Rani)
}

doi: $10.24200 /$ sci. 2019.53194 .3103 and modern treatment based on new techniques and tools, several uncertain cases related to DMP would arise so that decision-makers are no longer satisfied with the numerical values expressed in terms of crisp numbers. Therefore, to quantify different information into the analysis and analyze the information in a more accurate manner, many researchers have developed several types of algorithms using the theories of Fuzzy Set (FS) [1], Intuitionistic Fuzzy Set (IFS) [2], Linguistic Interval-Valued IFS (LIVIFS) [3], Complex FS (CFS) [4], Complex IFS (CIFS) [5], and complex interval-valued IFS [6]. In FS and IFS environments, information associated with each object is defined by Membership Degrees (MDs) and Non-Membership Degrees (NMDs) whose sum is not more than one. However, in LIVIFS, the required information was collected in the qualitative rather than quantitative form using linguistic variables. Further, in CFS and CIFS, information is practiced under the complex 
environment where the domain of IFS extended the domains of MDs and NMDs from the real set to the complex-valued set with a unit disc.

In such different environments, some researchers have put considerable effort into offering different methods and algorithms for solving MCDM problem in different fields through either Aggregation Operators (AOs) [7-14] or Information Measure (IM) [15-22]. For example, under the IFS environment, the weighted average and geometric AOs were explained in $[7,8]$. Further, Garg $[9,23]$ added to their explanation by incorporating the degree of hesitancy between the pairs of degrees. Some Hamacher AOs for IFSs were detailed by Huang [11] and Garg [24]. Apart from the AOs, the IMs play a significant role in treating imperfect and uncertain information. Of note, similarity, entropy, inclusion, etc. are the measures used by the researchers to examine the DMPs. To be more specific, similarity measures deal with two objects to compute the degree of similarity, entropy quantifies the degree of fuzziness in the set, and inclusion measures give the extent to which a set is contained in another set. On a separate plane, Divergence Measures (DvMs) are one of the most well-known tools for holding the uncertainty associated with the set. They depict the degree of discrimination between two objects. In the literature, all these measures have been greatly investigated as vital topics. For example, Kullback and Leibler [25] first introduced the concept of the DvM between two probability distributions. Later on, Bhandari and Pal [26] continued this measure to the FSs. Vlachos and Sergiadis [27] extended the idea of the DvM from FSs to IFSs. Zeng and $\mathrm{Li}$ [17] presented the correlation coefficients for IFSs. Garg [28] exhibited an improved cosine similarity measure for IFSs. Garg and Kumar [29] presented some similarity measures for IFSs based on the connection numbers of set pair analysis theory. Ohlan [30] presented the Intuitionistic Fuzzy (IF) exponential DvM along with its distinct properties and proposed a method for dealing with DMPs. Garg et al. [31] introduced parametric directed DvM under IFS theory to solve the DMPs. Mishra et al. [32] proposed Jensen-exponential DvM and the corresponding DMP under IFS environment. Furthermore, a number of researchers [33-37] have examined different IMs and applied them to DMPs.

From the earlier comprehensive studies and DMPs, it was perceived that their proposed approaches were restricted to some extent by handling only the uncertainty; however, they now fail to deal with the variations at a given phase of time in the data. The information gathered from the medical research, database for biometric and facial recognition, etc., regularly changes with the passage of time. Therefore, there is a demand to add the supplementary parameter into the study by representing this variation and, hence, handle the data accurately. In this respect, Ramot et al. [4] explained the concept of CFS by extending the domain of MDs from the real set to a complex-valued set with a unit disc. Further, to improve this theory, several properties such as complement, intersection, union, etc. were investigated by Ramot et al. [4,38]. Dick et al. [40] examined the association between the CFS and the Pythagorean FS [39]. A brief survey of the CFSs and logic was presented by Yazdanbakhsh and Dick [41]. Given their remarks, the degree of disagreement was not included in CFSs. Therefore, to properly evaluate the data, Alkouri and Salleh (2012) [5] stretched out the idea of CFS to CIFS by including the NMDs of the unit disc along the MDs into the analysis. Later on, some relations, projections, and measures for CIFSs were studied by Alkouri and Salleh [42]. Kumar and Bajaj [43] defined both entropy and distance measures for CIF soft sets. To further expand the CIFSs, Rani and Garg [44] presented the degree of dissimilarities among the CIFSs. Moreover, to measure the bond between CIFSs, Garg and Rani [45] defined correlation coefficients for them. Garg and Rani $[46,47]$ presented some averaging and geometric AOs for CIFSs. Quran and Hassan [48] suggested the required operations for complex neutrosophic soft sets. Rani and Garg [49] proposed power AOs for group DMPs under CIFS environment. Garg and Rani [50] presented some generalized Bonferroni mean AOs for CIFSs using Archimedean $t$-norm operations. Recently, Garg and Rani [51] presented exponential, logarithmic, and compensative AOs to aggregate different CIFSs. Also, Garg and Rani [52] studied different IMs of CIFSs.

The CIFS is a generalization of the IFS, taking into account both MDs and NMDs on the complex argument plane. Here, the amplitude term gives the extent of belongingness, and the phase term represents the periodicity of an object. Clearly, these phase terms distinguish the CIFS from the traditional IFS theory. In IFS theory, the factor of periodicity is completely ignored and hence, there is a certain loss of information. To avoid such a loss, the factor of periodicity was included in the analysis. To further illustrate the concept of phase terms, consider a certain company who wants to purchase cars from the carmakers regarding the features such as: (i) models and (ii) production dates of cars. Since the carmakers produce the same models of cars with slight improvements and differences every year, people, aware of the changes made, go along with the new model regardless of their judgments. In this regard, the production date of cars plays a significant role during the purchasing or decision. Therefore, such a two-dimensional problem cannot be simultaneously modeled in both existing FSs or IFSs environments. Furthermore, in order to execute such types of problems under IFS environment, first, two or more IFSs should be taken into account by the decision- 
Table 1. Comparison of Complex IFS (CIFS) model with existing models in literature

\begin{tabular}{lccccc}
\hline Features & Uncertainty & Falsity & Hesitation & Periodicity & $\begin{array}{c}\text { Ability to represent two- } \\
\text { dimensional information }\end{array}$ \\
\hline FS & $\checkmark$ & $\times$ & $\times$ & $\times$ & $\times$ \\
IVFS & $\checkmark$ & $\times$ & $\times$ & $\times$ & $\times$ \\
IFS & $\checkmark$ & $\checkmark$ & $\checkmark$ & $\times$ & $\times$ \\
IVIFS & $\checkmark$ & $\checkmark$ & $\checkmark$ & $\times$ & $\checkmark$ \\
CFS & $\checkmark$ & $\times$ & $\times$ & $\checkmark$ & $\checkmark$ \\
IVCFS & $\checkmark$ & $\times$ & $\times$ & $\checkmark$ & $\checkmark$ \\
CIFS & $\checkmark$ & $\checkmark$ & $\checkmark$ & $\checkmark$ & $\times$ \\
\hline
\end{tabular}

makers, thus leading to an increase in the execution time and number of computations while solving the problem. However, CIFS is a better representation of such problems in which both dimensions are considered as a single set. In addition, it is a better representation of the data than the existing ones. The salient features of CIFSs over several existing sets are given in Table 1.

Due to the complex DM process, it is inevitable to measure the degree of discrimination between the pairs of the sets. For this purpose, the IMs are the most efficient tools. Amongst the multiple measures such as entropy, similarity, inclusion, etc., the DvMs have the quality to evaluate the discrimination degree between the sets. Thus, encouraged from the hallmarks of the CIFS model and quality of DvM, the main objective of this study is to develop some exponentialbased DvMs to quantify the information. To this end, the information was designated under the CIFS model to quantify the data using the proposed measure for solving the DMPs. Some axioms and their properties are discussed in detail. Later, based on the intended study, an algorithm was elaborated to solve the DMPs and illustrate them through several numerical examples. To the best of the authors' knowledge, no study has been conducted so far on DvM and their impact on DMPs under the CIFS study. In this regard, a necessity was felt to scrutinize it under the environment and control its impact on the DM process. Accordingly, based on the advantages of the CIFSs model, the chief contributions of this research work are classified into three parts:

1. To propose the exponential DvM to measure the discrimination between the pairs of CIFSs;

2. To establish DM approach using the proposed measures;

3. To demonstrate the developed method with several examples and showcase its feasibility.

The rest of this paper is organized as follows. Section 2 introduces the basic concepts of IFSs and CIFSs. Section 3 introduces the concept of DvMs for CIFSs and examines their properties. Section 4 evaluates the function of maximizing deviation method to determine the weights, followed by the DM approach to MCDM problem. Section 5 clarifies the approach with some useful models. Finally, Section 6 presents the concluding remarks.

\section{Preliminaries}

Let $\mathcal{X}$ be the universal set. Here, we have reviewed the basic definitions related to IFSs and CIFSs here.

Definition 2.1 [1]. A FS $\mathcal{F}$ on $\mathcal{X}$ is defined as follows:

$$
\mathcal{F}=\left\{\left\langle x, u_{\mathcal{F}}(x)\right\rangle \mid x \in \mathcal{X}\right\},
$$

where $u_{\mathcal{F}}(x) \in[0,1]$ represents the MD of element $x$.

Definition 2.2 [25]. The degree of discrimination, known as DvM, between two discrete distributions $\mathcal{P}=\left(p_{1}, p_{2}, \cdots, p_{n}\right)$ and $\mathcal{Q}=\left(q_{1}, q_{2}, \cdots, q_{n}\right)$ is given as follows:

$$
D(\mathcal{P}, \mathcal{Q})=\sum_{j=1}^{n} p_{j} \log \left(\frac{p_{j}}{q_{j}}\right) .
$$

Definition 2.3 [26]. For two FSs $\mathcal{F}=\left\{\left(x, u_{\mathcal{F}}(x)\right)\right.$ $x \in \mathcal{X}\}$ and $\mathcal{G}=\left\{\left(x, u_{\mathcal{G}}(x)\right) \mid x \in \mathcal{X}\right\}$, the fuzzy DvM is defined as:

$$
\begin{aligned}
D(\mathcal{F}, \mathcal{G})= & \frac{1}{n} \sum_{j=1}^{n}\left[u_{\mathcal{F}}\left(x_{j}\right) \log \left(\frac{u_{\mathcal{F}}\left(x_{j}\right)}{u_{\mathcal{G}}\left(x_{j}\right)}\right)\right. \\
& \left.+\left(1-u_{\mathcal{F}}\left(x_{j}\right)\right) \log \left(\frac{1-u_{\mathcal{F}}\left(x_{j}\right)}{1-u_{\mathcal{G}}\left(x_{j}\right)}\right)\right]
\end{aligned}
$$

Definition 2.4 [2]. An IFS $\mathcal{I}$ on $\mathcal{X}$ is defined as:

$$
\mathcal{I}=\left\{\left\langle x, u_{\mathcal{I}}(x), v_{\mathcal{I}}(x)\right\rangle \mid x \in \mathcal{X}\right\}
$$

where $u_{\mathcal{I}}, v_{\mathcal{I}}: \mathcal{X} \rightarrow[0,1]$ represent the MD and NMD functions of $x$ to $\mathcal{I}$, respectively, such that $u_{\mathcal{I}}(x)+$ $v_{\mathcal{I}}(x) \leq 1$ for each $x$.

Definition 2.5 [27]. For two IFSs $\mathcal{I}=\left\{\left(x, u_{\mathcal{I}}\right.\right.$ $\left.\left.(x), v_{\mathcal{I}}(x)\right) \mid x \in \mathcal{X}\right\}$ and $\mathcal{J}=\left\{\left(x, u_{\mathcal{J}}(x), v_{\mathcal{J}}(x)\right) \mid\right.$ 
$x \in \mathcal{X}\}$ defined on $\mathcal{X}$, the DvM of $\mathcal{I}$ relative to $\mathcal{J}$ is defined as:

$$
\begin{aligned}
D(\mathcal{I}, \mathcal{J})= & \frac{1}{n} \sum_{j=1}^{n}\left[u_{\mathcal{I}}\left(x_{j}\right) \log \left(\frac{2 u_{\mathcal{I}}\left(x_{j}\right)}{u_{\mathcal{I}}\left(x_{j}\right)+u_{\mathcal{J}}\left(x_{j}\right)}\right)\right. \\
& \left.+v_{\mathcal{I}}\left(x_{j}\right) \log \left(\frac{2 v_{\mathcal{I}}\left(x_{j}\right)}{v_{\mathcal{I}}\left(x_{j}\right)+v_{\mathcal{J}}\left(x_{j}\right)}\right)\right] .
\end{aligned}
$$

Later, Garg et al. [31] defined some generalized parametric divergence of order $\alpha$ and degree $\beta$ under IFS environment obtained by Eq. (6) as shown in Box I.

Definition 2.6 [4]. A CFS $\mathcal{K}$ defined on $\mathcal{X}$ is given as:

$$
\mathcal{K}=\left\{\left(x, \mu_{\mathcal{K}}(x)\right): x \in \mathcal{X}\right\}
$$

where $\mu_{\mathcal{K}}: \mathcal{X} \rightarrow\{a: a \in C,|a| \leq 1\}$ is a complexvalued MD function and defined by:

$$
\mu_{\mathcal{K}}(x)=r_{\mathcal{K}}(x) e^{i 2 \pi w_{r_{\mathcal{K}}}(x)},
$$

where $i=\sqrt{-1}, 0 \leq r_{\mathcal{K}}(x), w_{r_{\mathcal{K}}}(x) \leq 1$.

Definition 2.7 [5]. A CIFS $\mathcal{K}$ on $\mathcal{X}$ is given as:

$$
\mathcal{K}=\left\{\left(x, \mu_{\mathcal{K}}(x), \gamma_{\mathcal{K}}(x)\right): x \in \mathcal{X}\right\}
$$

where $\mu_{\mathcal{K}}$ and $\gamma_{\mathcal{K}}$ are the complex-valued MD and NMD functions defined as $\mu_{\mathcal{K}}(x)=r_{\mathcal{K}}(x) e^{2 \pi i w_{r_{\mathcal{K}}}(x)}$ and $\gamma_{\mathcal{K}}(x)=k_{\mathcal{K}}(x) e^{2 \pi i w_{k_{\mathcal{K}}}(x)}$, where $0 \leq r_{\mathcal{K}}(x)$,
$k_{\mathcal{K}}(x) \leq 1 ; 0 \leq r_{\mathcal{K}}(x)+k_{\mathcal{K}}(x) \leq 1$ and $0 \leq$ $w_{r_{\mathcal{K}}}(x), w_{k_{\mathcal{K}}}(x), w_{r_{\mathcal{K}}}(x)+w_{k_{\mathcal{K}}}(x) \leq 1$. In this study, such a pair is regarded as $\mathcal{K}=\left(\left(r_{\mathcal{K}}, w_{r_{\mathcal{K}}}\right),\left(k_{\mathcal{K}}, w_{k_{\mathcal{K}}}\right)\right)$, which is called Complex Intuitionistic Fuzzy Number (CIFN).

Definition 2.8 [5]. Let $\mathcal{K}=\left\{\left(\left(r_{\mathcal{K}}(x), w_{r_{\mathcal{K}}}(x)\right)\right.\right.$, $\left.\left.\left(k_{\mathcal{K}}(x), w_{k_{\mathcal{K}}}(x)\right)\right): x \in \mathcal{X}\right\}$ and $\mathcal{M}=\left\{\left(\left(r_{\mathcal{M}}(x)\right.\right.\right.$, $\left.\left.\left.w_{r_{\mathcal{M}}}(x)\right),\left(k_{\mathcal{M}}(x), w_{k_{\mathcal{M}}}(x)\right)\right): x \in \mathcal{X}\right\}$ be two CIFSs. Then, we define:

(i) $\mathcal{K} \subseteq \mathcal{M}$ if $r_{\mathcal{K}}(x) \leq r_{\mathcal{M}}(x), k_{\mathcal{K}}(x) \geq k_{\mathcal{M}}(x)$ and $w_{r_{\mathcal{K}}}(x) \leq w_{r_{\mathcal{M}}}(x), w_{k_{\mathcal{K}}}(x) \geq w_{k_{\mathcal{M}}}(x)$;

(ii) $\mathcal{K}=\mathcal{M} \Leftrightarrow \mathcal{K} \subseteq \mathcal{M}$ and $\mathcal{M} \subseteq \mathcal{K}$;

(iii) $\mathcal{K}^{c}=\left\{\left(\left(k_{\mathcal{K}}(x), w_{k_{\mathcal{K}}}(x)\right),\left(r_{\mathcal{K}}(x), w_{r_{\mathcal{K}}}(x)\right)\right): x \in\right.$ $\mathcal{X}\}$

(iv) $\mathcal{K} \cup \mathcal{M}=\left\{\left(\left(r_{\mathcal{K} \cup \mathcal{M}}(x), w_{r_{\mathcal{K} \cup \mathcal{M}}}(x)\right),\left(k_{\mathcal{K} \cup \mathcal{M}}(x)\right.\right.\right.$, $\left.\left.\left.w_{k_{\mathcal{K} \cup \mathcal{M}}}(x)\right)\right): x \in \mathcal{X}\right\}$ where $r_{\mathcal{K} \cup \mathcal{M}}(x)=$ $\max \left\{r_{\mathcal{K}}(x), r_{\mathcal{M}}(x)\right\}, \quad k_{\mathcal{K} \cup \mathcal{M}}(x)=\min \left\{k_{\mathcal{K}}(x)\right.$, $\left.k_{\mathcal{M}}(x)\right\}, w_{r_{\mathcal{K} \cup \mathcal{M}}}(x)=\max \left\{w_{r_{\mathcal{K}}}(x), w_{r_{\mathcal{M}}}(x)\right\}$ and $w_{k_{\mathcal{K} \cup \mathcal{M}}}(x)=\min \left\{w_{k_{\mathcal{K}}}(x), w_{k_{\mathcal{M}}}(x)\right\}$;

(v) $\mathcal{K} \cap \mathcal{M}=\left\{\left(\left(r_{\mathcal{K} \cap \mathcal{M}}(x), w_{r_{\mathcal{K} \cap \mathcal{M}}}(x)\right),\left(k_{\mathcal{K} \cap \mathcal{M}}(x)\right.\right.\right.$, $\left.\left.\left.w_{k_{\mathcal{K} \cap \mathcal{M}}}(x)\right)\right): x \in \mathcal{X}\right\}$ where $r_{\mathcal{K} \cap \mathcal{M}}(x)=$ $\min \left\{r_{\mathcal{K}}(x), r_{\mathcal{M}}(x)\right\}, \quad k_{\mathcal{K} \cap \mathcal{M}}(x)=\max \left\{k_{\mathcal{K}}(x)\right.$, $\left.k_{\mathcal{M}}(x)\right\}, w_{r_{\mathcal{K} \cap \mathcal{M}}}(x)=\min \left\{w_{r_{\mathcal{K}}}(x), w_{r_{\mathcal{M}}}(x)\right\}$ and $w_{k_{\mathcal{K} \cap \mathcal{M}}}(x)=\max \left\{w_{k_{\mathcal{K}}}(x), w_{k_{\mathcal{M}}}(x)\right\}$.

$$
D(\mathcal{I}, \mathcal{J})=\frac{\alpha}{n(2-\beta)} \sum_{j=1}^{n}\left[\begin{array}{c}
u_{\mathcal{I}}^{\frac{\alpha}{2-\beta}}\left(x_{j}\right) \log \left(\frac{u_{\mathcal{I}}^{\frac{\alpha}{2-\beta}}\left(x_{j}\right)}{\lambda u_{\mathcal{I}}^{\frac{\alpha}{2-\beta}}\left(x_{j}\right)+(1-\lambda) u_{\mathcal{J}}^{\frac{\alpha}{2-\beta}}\left(x_{j}\right)}\right) \\
+v_{\mathcal{I}}^{\frac{\alpha}{2-\beta}}\left(x_{j}\right) \log \left(\frac{v_{\mathcal{I}}^{\frac{\alpha}{2-\beta}}\left(x_{j}\right)}{\lambda v_{\mathcal{I}}^{\frac{\alpha}{2-\beta}}\left(x_{j}\right)+(1-\lambda) v_{\mathcal{J}}^{\frac{\alpha}{2-\beta}}\left(x_{j}\right)}\right) \\
+h_{\mathcal{I}}^{\frac{\alpha}{2-\beta}}\left(x_{j}\right) \log \left(\frac{h_{\mathcal{I}}^{\frac{\alpha}{2-\beta}}\left(x_{j}\right)}{\lambda h_{\mathcal{I}}^{\frac{\alpha}{2-\beta}}\left(x_{j}\right)+(1-\lambda) h_{\mathcal{J}}^{\frac{\alpha}{2-\beta}}\left(x_{j}\right)}\right)
\end{array}\right]
$$




\section{Proposed exponential divergence measure}

Let $\Phi(\mathcal{X})$ be the class of CIFSs. Then, here, we define the exponential DvM for $\Phi(\mathcal{X})$ and study their properties:

Definition 3.1. For $\mathcal{K}, \mathcal{M} \in \Phi(\mathcal{X})$, a real function $\mathcal{D} v: \Phi(\mathcal{X}) \times \Phi(\mathcal{X}) \rightarrow R^{+}$is called a DvM, if:

(P1) $\mathcal{D} v(\mathcal{K}, \mathcal{M}) \geq 0$

(P2) $\mathcal{D} v(\mathcal{K}, \mathcal{M})=\mathcal{D} v(\mathcal{M}, \mathcal{K})$;

(P3) $\mathcal{D} v(\mathcal{K}, \mathcal{M})=0$ if $\mathcal{K}=\mathcal{M}$;

(P4) $\mathcal{D} v(\mathcal{K}, \mathcal{M})=\mathcal{D} v\left(\mathcal{K}^{c}, \mathcal{M}^{c}\right)$.

Definition 3.2. For two CIFSs $\mathcal{K}=\left\{\left(\left(r_{\mathcal{K}}(x), w_{r_{\mathcal{K}}}(x)\right)\right.\right.$, $\left.\left.\left(k_{\mathcal{K}}(x), w_{k_{\mathcal{K}}}(x)\right)\right): x \in \mathcal{X}\right\}$ and $\mathcal{M}=\left\{\left(\left(r_{\mathcal{M}}(x), w_{r_{\mathcal{M}}}\right.\right.\right.$ $\left.\left.(x)),\left(k_{\mathcal{M}}(x), w_{k_{\mathcal{M}}}(x)\right)\right): x \in \mathcal{X}\right\}$, the degree of discrimination between them is defined by Eq. (9) as shown in Box II, where 'exp' refers to the exponential function.

Here, $E(\mathcal{K}, \mathcal{M}) \neq E\left(\mathcal{K}^{c}, \mathcal{M}^{c}\right)$. Obviously, the degree of discriminations of $\mathcal{K}$ from $\mathcal{M}$ and $\mathcal{K}^{c}$ from $\mathcal{M}^{c}$ should be the same. In order to imbue the measure with symmetry, we define the symmetric DvM as follows.

Definition 3.3. A symmetric exponential divergence measure for two CIFSs $\mathcal{K}$ and $\mathcal{M}$, denoted by $\mathcal{D} v(\mathcal{K}, \mathcal{M})$ is defined as follows:

$\mathcal{D} v(\mathcal{K}, \mathcal{M})=E(\mathcal{K}, \mathcal{M})+E\left(\mathcal{K}^{c}, \mathcal{M}^{c}\right)$

$$
\begin{aligned}
= & \frac{1}{4 n\left(1-e^{-1}\right)} \sum_{j=1}^{n} \\
& {\left[\begin{array}{c}
4-\left(1-t_{j}(\mathcal{K}, \mathcal{M})\right) \exp \left(t_{j}(\mathcal{K}, \mathcal{M})\right) \\
-\left(1+t_{j}(\mathcal{K}, \mathcal{M})\right) \exp \left(-t_{j}(\mathcal{K}, \mathcal{M})\right) \\
-\left(1-s_{j}(\mathcal{K}, \mathcal{M})\right) \exp \left(s_{j}(\mathcal{K}, \mathcal{M})\right) \\
-\left(1+s_{j}(\mathcal{K}, \mathcal{M})\right) \exp \left(-s_{j}(\mathcal{K}, \mathcal{M})\right)
\end{array}\right](10) }
\end{aligned}
$$

where:

$$
\begin{aligned}
& t_{j}(\mathcal{K}, \mathcal{M}) \\
& \quad=\frac{\left(r_{\mathcal{K}}\left(x_{j}\right)-r_{\mathcal{M}}\left(x_{j}\right)\right)-\left(k_{\mathcal{K}}\left(x_{j}\right)-k_{\mathcal{M}}\left(x_{j}\right)\right)}{2},
\end{aligned}
$$

and:

$$
\begin{aligned}
& s_{j}(\mathcal{K}, \mathcal{M}) \\
& \quad=\frac{\left(w_{r_{\mathcal{K}}}\left(x_{j}\right)-w_{r_{\mathcal{M}}}\left(x_{j}\right)\right)-\left(w_{k_{\mathcal{K}}}\left(x_{j}\right)-w_{k_{\mathcal{M}}}\left(x_{j}\right)\right)}{2} .
\end{aligned}
$$

Remark 3.1. $t_{j}(\mathcal{K}, \mathcal{M})=-t_{j}(\mathcal{M}, \mathcal{K})$ and $s_{j}(\mathcal{K}, \mathcal{M})$ $=-s_{j}(\mathcal{M}, \mathcal{K})$.

Before proving that Eq. (10) is valid DvM, the two following lemmas can be stated as follows.

Lemma 3.1. Let $f(y)=2-(1-y) \exp (y)-(1+$ $y) \exp (-y)$ be a function, where $y \in[-1,1]$. Then:

$$
0 \leq f(y) \leq 2-2 \exp (-1) \text {. }
$$

Proof. Since $f(y)=2-(1-y) \exp (y)-(1+y) \exp (-y)$. $f^{\prime}(y)=y(\exp (y)+\exp (-y))$ which follows that $f(y)$ is decreasing in $[-1,0]$ and increasing in $[0,1]$. Therefore, when $y \in[-1,0], f(0) \leq f(y) \leq f(-1)$ i.e., $0 \leq f(y) \leq$ $2-2 \exp (-1)$ and similarly for $y \in[0,1], f(0) \leq f(y) \leq$ $f(1)$ i.e., $0 \leq f(y) \leq 2-2 \exp (-1)$. Hence, for $y \in$ $[-1,1]$, we have $0 \leq f(y) \leq 2-2 \exp (-1)$.

Lemma 3.2. For $y \in[-1,0]$, the functions $f_{1}(y)=$ $(1-y) \exp (y)$ and $f_{2}(y)=(1+y) \exp (-y)$ are the increasing functions.

Proof. Since $f_{1}(y)=(1-y) \exp (y)$. For $y \in[-1,0]$, $f_{1}^{\prime}(y)=-y \exp (y) \geq 0$ implying that $f_{1}(y)$ is an increasing function. Similarly, we can prove that $f_{2}(y)$ is an increasing function for $y \in[-1,0]$.

Theorem 3.1. The measure presented in Definition 3.3 is a valid divergence measure.

$$
E(\mathcal{K}, \mathcal{M})=\frac{1}{4 n\left(1-e^{-1}\right)} \sum_{j=1}^{n}\left[\begin{array}{l}
2-\left(\frac{k_{\mathcal{K}}\left(x_{j}\right)+1-r_{\mathcal{K}}\left(x_{j}\right)}{2}\right) \exp \left(\frac{\left(r_{\mathcal{K}}\left(x_{j}\right)-r_{\mathcal{M}}\left(x_{j}\right)\right)-\left(k_{\mathcal{K}}\left(x_{j}\right)-k_{\mathcal{M}}\left(x_{j}\right)\right)}{2}\right) \\
-\left(\frac{r_{\mathcal{K}}\left(x_{j}\right)+1-k_{\mathcal{K}}\left(x_{j}\right)}{2}\right) \exp \left(\frac{\left(r_{\mathcal{M}}\left(x_{j}\right)-r_{\mathcal{K}}\left(x_{j}\right)\right)-\left(k_{\mathcal{M}}\left(x_{j}\right)-k_{\mathcal{K}}\left(x_{j}\right)\right)}{2}\right) \\
-\left(\frac{\left.w_{k_{\mathcal{K}}\left(x_{j}\right)}+1-w_{r_{\mathcal{K}}\left(x_{j}\right)}\right)}{2}\right) \exp \left(\frac{\left(w_{r_{\mathcal{K}}\left(x_{j}\right)}-w_{r_{\mathcal{M}}\left(x_{j}\right)}\right)-\left(w_{k_{\mathcal{K}}\left(x_{j}\right)}-w_{k_{\mathcal{M}}\left(x_{j}\right)}\right)}{2}\right) \\
-\left(\frac{\left.w_{r_{\mathcal{K}}\left(x_{j}\right)}+1-w_{k_{\mathcal{K}}\left(x_{j}\right)}\right)}{2}\right) \exp \left(\frac{\left(w_{r_{\mathcal{M}}\left(x_{j}\right)}-w_{r_{\mathcal{K}}\left(x_{j}\right)}\right)-\left(w_{k_{\mathcal{M}}\left(x_{j}\right)}-w_{k_{\mathcal{K}}\left(x_{j}\right)}\right)}{2}\right)
\end{array}\right]
$$


Proof. Let $\mathcal{K}=\left\{\left(\left(r_{\mathcal{K}}(x), w_{r_{\mathcal{K}}}(x)\right),\left(k_{\mathcal{K}}(x), w_{k_{\mathcal{K}}}(x)\right)\right)\right.$ : $x \in \mathcal{X}\}, \mathcal{M}=\left\{\left(\left(r_{\mathcal{M}}(x), w_{r_{\mathcal{M}}}(x)\right),\left(k_{\mathcal{M}}(x), w_{k_{\mathcal{M}}}(x)\right)\right):\right.$ $x \in \mathcal{X}\}$ and $\mathcal{N}=\left\{\left(\left(r_{\mathcal{N}}(x), w_{r_{\mathcal{N}}}(x)\right),\left(k_{\mathcal{N}}(x), w_{k_{\mathcal{N}}}\right.\right.\right.$ $(x))): x \in \mathcal{X}\}$ be three CIFSs. Then, to prove the results, we need to show that Eq. (10) satisfies the following axioms:

(P1) $0 \leq \mathcal{D} v(\mathcal{K}, \mathcal{M}) \leq 1$

$(\mathrm{P} 2) \mathcal{D} v(\mathcal{K}, \mathcal{M})=0$ if $\mathcal{K}=\mathcal{M}$;

(P3) $\mathcal{D} v(\mathcal{K}, \mathcal{M})=\mathcal{D} v(\mathcal{M}, \mathcal{K})$;

(P4) If $\mathcal{K} \subseteq \mathcal{M} \subseteq \mathcal{N}$ then, $\mathcal{D} v(\mathcal{K}, \mathcal{N}) \geq \mathcal{D} v(\mathcal{K}, \mathcal{M})$ and $\mathcal{D} v(\mathcal{K}, \mathcal{N}) \geq \mathcal{D} v(\mathcal{M}, \mathcal{N})$.

By defining CIFSs, we have:

(P1) Since $0 \leq r_{\mathcal{K}}\left(x_{j}\right), r_{\mathcal{M}}\left(x_{j}\right), k_{\mathcal{K}}\left(x_{j}\right), k_{\mathcal{M}}\left(x_{j}\right) \leq 1$. It implies that $-1 \leq r_{\mathcal{K}}\left(x_{j}\right)-r_{\mathcal{M}}\left(x_{j}\right) \leq 1$; $-1 \leq k_{\mathcal{K}}\left(x_{j}\right)-k_{\mathcal{M}}\left(x_{j}\right) \leq 1$ which gives that $-2 \leq\left(r_{\mathcal{K}}\left(x_{j}\right)-r_{\mathcal{M}}\left(x_{j}\right)\right)-\left(k_{\mathcal{K}}\left(x_{j}\right)-k_{\mathcal{M}}\left(x_{j}\right)\right) \leq 2$ and hence, $-1 \leq t_{j}(\mathcal{K}, \mathcal{M}) \leq 1$. Similarly, we can prove that, $-1 \leq s_{j}(\mathcal{K}, \mathcal{M}) \leq 1$. Then, using the above Lemma 3.1 , we obtain that $0 \leq 2-\left(1-t_{j}(\mathcal{K}, \mathcal{M})\right) \exp \left(t_{j}(\mathcal{K}, \mathcal{M})\right)-(1+$ $\left.t_{j}(\mathcal{K}, \mathcal{M})\right) \exp \left(-t_{j}(\mathcal{K}, \mathcal{M})\right) \leq 2-2 \exp (-1)$ and $0 \leq 2-\left(1-s_{j}(\mathcal{K}, \mathcal{M})\right) \exp \left(s_{j}(\mathcal{K}, \mathcal{M})\right)-(1+$ $\left.s_{j}(\overline{\mathcal{K}}, \mathcal{M})\right) \exp \left(-s_{j}(\mathcal{K}, \mathcal{M})\right) \leq 2-2 \exp (-1)$ which gives that $0 \leq 4-\left(1-t_{j}(\mathcal{K}, \mathcal{M})\right) \exp$ $\left(t_{j}(\mathcal{K}, \mathcal{M})\right)-\left(1+t_{j}(\mathcal{K}, \mathcal{M})\right) \exp \left(-t_{j}(\mathcal{K}, \mathcal{M})\right)$ $-\left(1-s_{j}(\mathcal{K}, \mathcal{M})\right) \exp \left(s_{j}(\mathcal{K}, \mathcal{M})\right)-\left(1+s_{j}(\mathcal{K}, \mathcal{M})\right)$ $\exp \left(-s_{j}(\mathcal{K}, \mathcal{M})\right) \leq 4-4 \exp (-1)$. Hence, $0 \leq$ $\mathcal{D} v(\mathcal{K}, \mathcal{M}) \leq 1$

(P2) For $\mathcal{K}=\mathcal{M}$, we have $r_{\mathcal{K}}\left(x_{j}\right)=r_{\mathcal{M}}\left(x_{j}\right), k_{\mathcal{K}}\left(x_{j}\right)=$ $k_{\mathcal{M}}\left(x_{j}\right), w_{r_{\mathcal{K}}}\left(x_{j}\right)=w_{r_{\mathcal{M}}}\left(x_{j}\right)$ and $w_{k_{\mathcal{K}}}\left(x_{j}\right)=$ $w_{k_{\mathcal{M}}}\left(x_{j}\right)$ for all $j$ 's which gives that $t_{j}(\mathcal{K}, \mathcal{M})=$ $s_{j}(\mathcal{K}, \mathcal{M})=0$. Thus, $\mathcal{D} v(\mathcal{K}, \mathcal{M})=0$;

(P3) As, $t_{j}(\mathcal{K}, \mathcal{M})=-t_{j}(\mathcal{M}, \mathcal{K})$ and $s_{j}(\mathcal{K}, \mathcal{M})=$ $-s_{j}(\mathcal{M}, \mathcal{K})$. Therefore, it follows that $\mathcal{D} v(\mathcal{K}, \mathcal{M})=\mathcal{D} v(\mathcal{M}, \mathcal{K})$

(P4) Since $\mathcal{K} \subseteq \mathcal{M} \subseteq \mathcal{N}$, it implies that $r_{\mathcal{K}}\left(x_{j}\right) \leq$ $r_{\mathcal{M}}\left(x_{j}\right) \leq r_{\mathcal{N}}\left(x_{j}\right)$ and $k_{\mathcal{K}}\left(x_{j}\right) \geq k_{\mathcal{M}}\left(x_{j}\right) \geq$ $k_{\mathcal{N}}\left(x_{j}\right)$ which gives that $r_{\mathcal{K}}\left(x_{j}\right)-r_{\mathcal{N}}\left(x_{j}\right) \leq$ $r_{\mathcal{K}}\left(x_{j}\right)-r_{\mathcal{M}}\left(x_{j}\right) \leq 0$ and $0 \leq k_{\mathcal{K}}\left(x_{j}\right)-k_{\mathcal{M}}$ $\left(x_{j}\right) \leq k_{\mathcal{K}}\left(x_{j}\right)-k_{\mathcal{N}}\left(x_{j}\right)$. It follows that $t_{j}(\mathcal{K}$, $\mathcal{N}) \leq t_{j}(\mathcal{K}, \mathcal{M}) \leq 0$. Also, $t_{j}(\mathcal{K}, \mathcal{N}), t_{j}(\mathcal{K}, \mathcal{M}) \geq$ -1. Then, by using Lemma 3.2 , we have $\left(1-t_{j}(\mathcal{K}, \mathcal{N})\right) \exp \left(t_{j}(\mathcal{K}, \mathcal{N})\right) \leq\left(1-t_{j}(\mathcal{K}, \mathcal{M})\right)$ $\exp \left(t_{j}(\mathcal{K}, \mathcal{M})\right)$ and $\left(1+t_{j}(\mathcal{K}, \mathcal{N})\right) \exp \left(-t_{j}\right.$ $(\mathcal{K}, \mathcal{N})) \leq\left(1+t_{j}(\mathcal{K}, \mathcal{M})\right) \exp \left(-t_{j}(\mathcal{K}, \mathcal{M})\right)$ Similarly, we can prove that $\left(1-s_{j}(\mathcal{K}, \mathcal{N})\right)$ $\exp \left(s_{j}(\mathcal{K}, \mathcal{N})\right) \leq\left(1-s_{j}(\mathcal{K}, \mathcal{M})\right) \exp \left(s_{j}(\mathcal{K}, \mathcal{M})\right)$ and $\left(1+s_{j}(\mathcal{K}, \mathcal{N})\right) \exp \left(-s_{j}(\mathcal{K}, \quad \mathcal{N})\right) \leq$ $\left(1+s_{j}(\mathcal{K}, \mathcal{M})\right) \exp \left(-s_{j}(\mathcal{K}, \mathcal{M})\right)$. Hence, $\mathcal{D} v$
$(\mathcal{K}, \mathcal{N}) \geq \mathcal{D} v(\mathcal{K}, \mathcal{M})$. Similarly, we can prove that $\mathcal{D} v(\mathcal{K}, \mathcal{N}) \geq \mathcal{D} v(\mathcal{M}, \mathcal{N})$.

The functionality of the proposed measure is clarified through the example below:

Example 3.1. For $\mathcal{X}=\left\{x_{1}, x_{2}, x_{3}\right\}$, let $\mathcal{K}=\left\{\left(x_{1},(0.4\right.\right.$, $0.1),(0.3,0.5)),\left(x_{2},(0.5,0.3),(0.1,0.2)\right),\left(x_{3},(0.7\right.$, $0.3),(0.2,0.3))\}$, and $\mathcal{M}=\left\{\left(x_{1},(0.6,0.3),(0.3,0.2)\right)\right.$, $\left.\left(x_{2},(0.4,0.3),(0.2,0.1)\right),\left(x_{3},(0.7,0.4),(0.1,0.2)\right)\right\}$ be two CIFSs. Then:

$$
\begin{aligned}
t_{1}(\mathcal{K}, \mathcal{M}) & =\frac{\left(r_{\mathcal{K}}\left(x_{1}\right)-r_{\mathcal{M}}\left(x_{1}\right)\right)-\left(k_{\mathcal{K}}\left(x_{1}\right)-k_{\mathcal{M}}\left(x_{1}\right)\right)}{2} \\
& =\frac{(0.4-0.6)-(0.3-0.3)}{2}=-0.1 .
\end{aligned}
$$

Similarly, $t_{2}(\mathcal{K}, \mathcal{M})=0.1, t_{3}(\mathcal{K}, \mathcal{M})=-0.05, s_{1}(\mathcal{K}$, $\mathcal{M})=-0.25, s_{2}(\mathcal{K}, \mathcal{M})=-0.05$, and $s_{3}(\mathcal{K}, \mathcal{M})=$ -0.1 can be obtained. Therefore, based on Eq. (10), we have:

$$
\left.\begin{array}{l}
\mathcal{D} v(\mathcal{K}, \mathcal{M})=\frac{1}{12\left(1-e^{-1}\right)} \\
-(1+0.25) e^{-0.25}-(1-0.25) e^{0.25} \\
+4-(1-0.1) e^{0.1}-(1+0.1) e^{-0.1} \\
-(1+0.05) e^{-0.05}-(1-0.05) e^{0.05} \\
+4-(1+0.05) e^{-0.05}-(1-0.05) e^{0.05} \\
-(1+0.1) e^{-0.1}-(1-0.1) e^{0.1}
\end{array}\right]
$$

In the following, we prove some propositions for the proposed divergence measure. For this, the universal set $\mathcal{X}$ was divided into two disjointed subsets $\mathcal{X}_{1}=$ $\left\{x_{j} \mid \mathcal{K}\left(x_{j}\right) \subseteq \mathcal{M}\left(x_{j}\right)\right\}$ and $\mathcal{X}_{2}=\left\{x_{j} \mid \mathcal{M}\left(x_{j}\right) \subseteq\right.$ $\left.\mathcal{K}\left(x_{j}\right)\right\}$. Then, the following propositions were satisfied based on these considerations.

Proposition 3.1. If $\mathcal{K}$ and $\mathcal{M}$ are two CIFSs defined on $\mathcal{X}$ such that they satisfy any $x_{j} \in \mathcal{X}$ either $\mathcal{K} \subseteq \mathcal{M}$ or $\mathcal{K} \supseteq \mathcal{M}$, then:

(i) $\mathcal{D} v(\mathcal{K} \cup \mathcal{M}, \mathcal{K} \cap \mathcal{M})=\mathcal{D} v(\mathcal{K}, \mathcal{M})$;

(ii) $\mathcal{D} v(\mathcal{K} \cap \mathcal{M}, \mathcal{K} \cup \mathcal{M})=\mathcal{D} v(\mathcal{K}, \mathcal{M})$.

Proof. Here, we prove Part (i) only, and Part (ii) can be similarly deduced. From Definition 3.3, we obtain the equation shown in Box III.

Proposition 3.2. For CIFSs $\mathcal{K}, \mathcal{M}$, and $\mathcal{N}$ defined on $\mathcal{X}$, we have: 


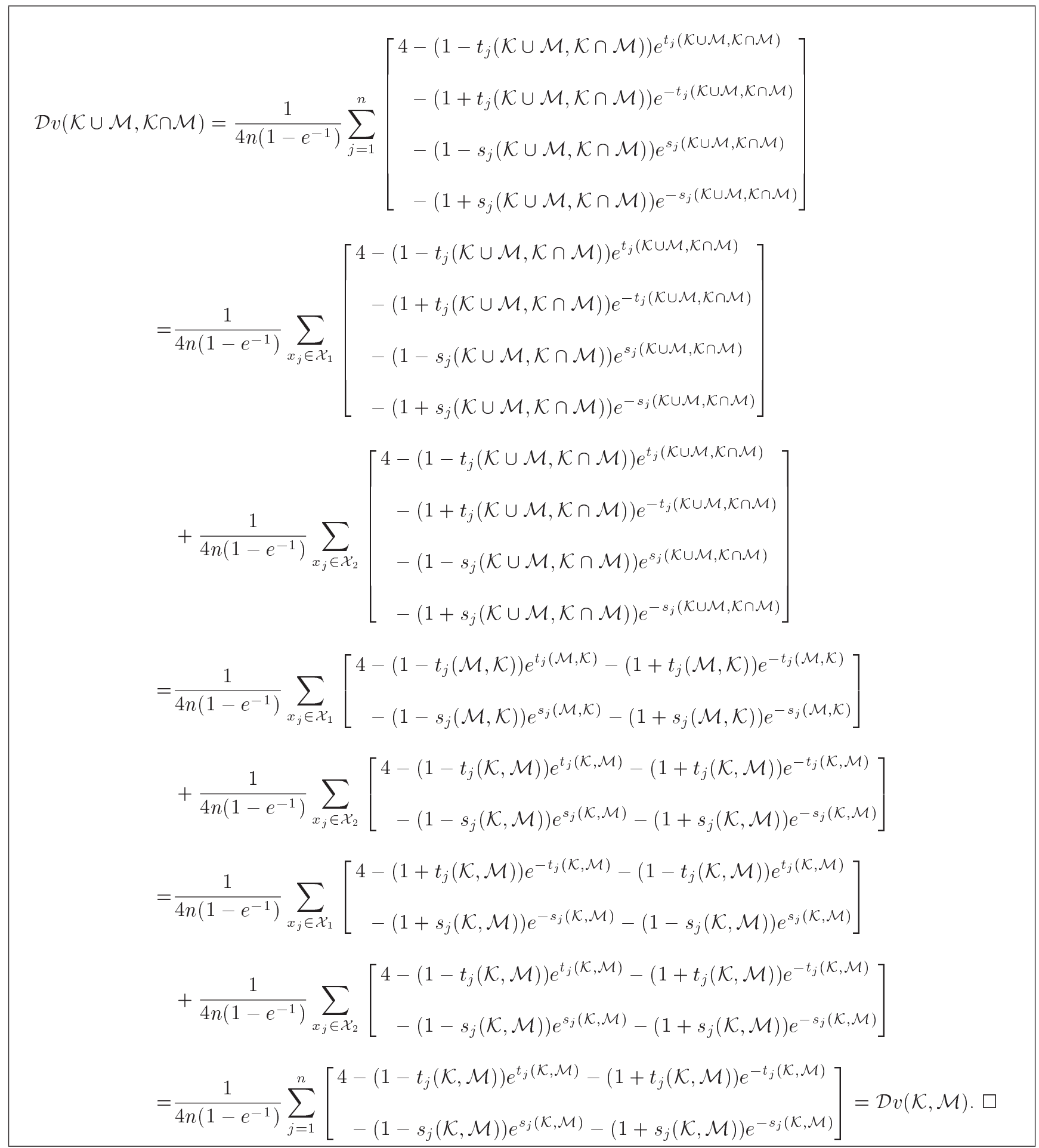

Box III

$\mathcal{D} v(\mathcal{K} \cup \mathcal{M}, \mathcal{N})+\mathcal{D} v(\mathcal{K} \cap \mathcal{M}, \mathcal{N})=\mathcal{D} v(\mathcal{K}, \mathcal{N})+\mathcal{D} v(\mathcal{M}, \mathcal{N})$

Proof. Through Definition 3.3, we obtain the equation shown in Box IV.

Proposition 3.3. For CIFSs $\mathcal{K}$ and $\mathcal{M}$ defined on $\mathcal{X}$, we have: (i) $\mathcal{D} v(\mathcal{K}, \mathcal{K} \cup \mathcal{M})+\mathcal{D} v(\mathcal{K}, \mathcal{K} \cap \mathcal{M})=\mathcal{D} v(\mathcal{K}, \mathcal{M})$;

(ii) $\mathcal{D} v(\mathcal{M}, \mathcal{K} \cup \mathcal{M})+\mathcal{D} v(\mathcal{M}, \mathcal{K} \cap \mathcal{M})=\mathcal{D} v(\mathcal{K}, \mathcal{M})$.

Proof. Considering $\mathcal{N}=\mathcal{K}$ in Proposition 3.2, we have $\mathcal{D} v(\mathcal{K} \cup \mathcal{M}, \mathcal{K})+\mathcal{D} v(\mathcal{K} \cap \mathcal{M}, \mathcal{K})=\mathcal{D} v(\mathcal{K}, \mathcal{K})+$ $\mathcal{D} v(\mathcal{M}, \mathcal{K})=\mathcal{D} v(\mathcal{K}, \mathcal{M})$. Hence, Part (i) holds and so does Part (ii). 


$$
\begin{aligned}
& \mathcal{D} v(\mathcal{K} \cup \mathcal{M}, \mathcal{N})+\mathcal{D} v(\mathcal{K} \cap \mathcal{M}, \mathcal{N}) \\
& =\frac{1}{4 n\left(1-e^{-1}\right)} \sum_{j=1}^{n}\left[\begin{array}{c}
4-\left(1-t_{j}(\mathcal{K} \cup \mathcal{M}, \mathcal{N})\right) e^{t_{j}(\mathcal{K} \cup \mathcal{M}, \mathcal{N})}-\left(1+t_{j}(\mathcal{K} \cup \mathcal{M}, \mathcal{N})\right) e^{-t_{j}(\mathcal{K} \cup \mathcal{M}, \mathcal{N})} \\
-\left(1-s_{j}(\mathcal{K} \cup \mathcal{M}, \mathcal{N})\right) e^{s_{j}(\mathcal{K} \cup \mathcal{M}, \mathcal{N})}-\left(1+s_{j}(\mathcal{K} \cup \mathcal{M}, \mathcal{N})\right) e^{-s_{j}(\mathcal{K} \cup \mathcal{M}, \mathcal{N})}
\end{array}\right] \\
& +\frac{1}{4 n\left(1-e^{-1}\right)} \sum_{j=1}^{n}\left[\begin{array}{c}
4-\left(1-t_{j}(\mathcal{K} \cap \mathcal{M}, \mathcal{N})\right) e^{t_{j}(\mathcal{K} \cap \mathcal{M}, \mathcal{N})}-\left(1+t_{j}(\mathcal{K} \cap \mathcal{M}, \mathcal{N})\right) e^{-t_{j}(\mathcal{K} \cap \mathcal{M}, \mathcal{N})} \\
-\left(1-s_{j}(\mathcal{K} \cap \mathcal{M}, \mathcal{N})\right) e^{s_{j}(\mathcal{K} \cap \mathcal{M}, \mathcal{N})}-\left(1+s_{j}(\mathcal{K} \cap \mathcal{M}, \mathcal{N})\right) e^{-s_{j}(\mathcal{K} \cap \mathcal{M}, \mathcal{N})}
\end{array}\right] \\
& =\frac{1}{4 n\left(1-e^{-1}\right)} \sum_{x_{j} \in \mathcal{X}_{1}}\left[\begin{array}{c}
4-\left(1-t_{j}(\mathcal{K} \cup \mathcal{M}, \mathcal{N})\right) e^{t_{j}(\mathcal{K} \cup \mathcal{M}, \mathcal{N})}-\left(1+t_{j}(\mathcal{K} \cup \mathcal{M}, \mathcal{N})\right) e^{-t_{j}(\mathcal{K} \cup \mathcal{M}, \mathcal{N})} \\
-\left(1-s_{j}(\mathcal{K} \cup \mathcal{M}, \mathcal{N})\right) e^{s_{j}(\mathcal{K} \cup \mathcal{M}, \mathcal{N})}-\left(1+s_{j}(\mathcal{K} \cup \mathcal{M}, \mathcal{N})\right) e^{-s_{j}(\mathcal{K} \cup \mathcal{M}, \mathcal{N})}
\end{array}\right] \\
& +\frac{1}{4 n\left(1-e^{-1}\right)} \sum_{x_{j} \in \mathcal{X}_{2}}\left[\begin{array}{c}
4-\left(1-t_{j}(\mathcal{K} \cup \mathcal{M}, \mathcal{N})\right) e^{t_{j}(\mathcal{K} \cup \mathcal{M}, \mathcal{N})}-\left(1+t_{j}(\mathcal{K} \cup \mathcal{M}, \mathcal{N})\right) e^{-t_{j}(\mathcal{K} \cup \mathcal{M}, \mathcal{N})} \\
-\left(1-s_{j}(\mathcal{K} \cup \mathcal{M}, \mathcal{N})\right) e^{s_{j}(\mathcal{K} \cup \mathcal{M}, \mathcal{N})}-\left(1+s_{j}(\mathcal{K} \cup \mathcal{M}, \mathcal{N})\right) e^{-s_{j}(\mathcal{K} \cup \mathcal{M}, \mathcal{N})}
\end{array}\right] \\
& +\frac{1}{4 n\left(1-e^{-1}\right)} \sum_{x_{j} \in \mathcal{X}_{1}}\left[\begin{array}{c}
4-\left(1-t_{j}(\mathcal{K} \cap \mathcal{M}, \mathcal{N})\right) e^{t_{j}(\mathcal{K} \cap \mathcal{M}, \mathcal{N})}-\left(1+t_{j}(\mathcal{K} \cap \mathcal{M}, \mathcal{N})\right) e^{-t_{j}(\mathcal{K} \cap \mathcal{M}, \mathcal{N})} \\
-\left(1-s_{j}(\mathcal{K} \cap \mathcal{M}, \mathcal{N})\right) e^{s_{j}(\mathcal{K} \cap \mathcal{M}, \mathcal{N})}-\left(1+s_{j}(\mathcal{K} \cap \mathcal{M}, \mathcal{N})\right) e^{-s_{j}(\mathcal{K} \cap \mathcal{M}, \mathcal{N})}
\end{array}\right] \\
& +\frac{1}{4 n\left(1-e^{-1}\right)} \sum_{x_{j} \in \mathcal{X}_{2}}\left[\begin{array}{c}
4-\left(1-t_{j}(\mathcal{K} \cap \mathcal{M}, \mathcal{N})\right) e^{t_{j}(\mathcal{K} \cap \mathcal{M}, \mathcal{N})}-\left(1+t_{j}(\mathcal{K} \cap \mathcal{M}, \mathcal{N})\right) e^{-t_{j}(\mathcal{K} \cap \mathcal{M}, \mathcal{N})} \\
-\left(1-s_{j}(\mathcal{K} \cap \mathcal{M}, \mathcal{N})\right) e^{s_{j}(\mathcal{K} \cap \mathcal{M}, \mathcal{N})}-\left(1+s_{j}(\mathcal{K} \cap \mathcal{M}, \mathcal{N})\right) e^{-s_{j}(\mathcal{K} \cap \mathcal{M}, \mathcal{N})}
\end{array}\right] \\
& =\frac{1}{4 n\left(1-e^{-1}\right)} \sum_{x_{j} \in \mathcal{X}_{1}}\left[\begin{array}{c}
4-\left(1-t_{j}(\mathcal{M}, \mathcal{N})\right) e^{t_{j}(\mathcal{M}, \mathcal{N})}-\left(1+t_{j}(\mathcal{M}, \mathcal{N})\right) e^{-t_{j}(\mathcal{M}, \mathcal{N})} \\
-\left(1-s_{j}(\mathcal{M}, \mathcal{N})\right) e^{s_{j}(\mathcal{M}, \mathcal{N})}-\left(1+s_{j}(\mathcal{M}, \mathcal{N})\right) e^{-s_{j}(\mathcal{M}, \mathcal{N})}
\end{array}\right] \\
& +\frac{1}{4 n\left(1-e^{-1}\right)} \sum_{x_{j} \in \mathcal{X}_{2}}\left[\begin{array}{c}
4-\left(1-t_{j}(\mathcal{K}, \mathcal{N})\right) e^{t_{j}(\mathcal{K}, \mathcal{N})}-\left(1+t_{j}(\mathcal{K}, \mathcal{N})\right) e^{-t_{j}(\mathcal{K}, \mathcal{N})} \\
-\left(1-s_{j}(\mathcal{K}, \mathcal{N})\right) e^{s_{j}(\mathcal{K}, \mathcal{N})}-\left(1+s_{j}(\mathcal{K}, \mathcal{N})\right) e^{-s_{j}(\mathcal{K}, \mathcal{N})}
\end{array}\right] \\
& +\frac{1}{4 n\left(1-e^{-1}\right)} \sum_{x_{j} \in \mathcal{X}_{1}}\left[\begin{array}{c}
4-\left(1-t_{j}(\mathcal{K}, \mathcal{N})\right) e^{t_{j}(\mathcal{K}, \mathcal{N})}-\left(1+t_{j}(\mathcal{K}, \mathcal{N})\right) e^{-t_{j}(\mathcal{K}, \mathcal{N})} \\
-\left(1-s_{j}(\mathcal{K}, \mathcal{N})\right) e^{s_{j}(\mathcal{K}, \mathcal{N})}-\left(1+s_{j}(\mathcal{K}, \mathcal{N})\right) e^{-s_{j}(\mathcal{K}, \mathcal{N})}
\end{array}\right] \\
& +\frac{1}{4 n\left(1-e^{-1}\right)} \sum_{x_{j} \in \mathcal{X}_{2}}\left[\begin{array}{c}
4-\left(1-t_{j}(\mathcal{M}, \mathcal{N})\right) e^{t_{j}(\mathcal{M}, \mathcal{N})}-\left(1+t_{j}(\mathcal{M}, \mathcal{N})\right) e^{-t_{j}(\mathcal{M}, \mathcal{N})} \\
-\left(1-s_{j}(\mathcal{M}, \mathcal{N})\right) e^{s_{j}(\mathcal{M}, \mathcal{N})}-\left(1+s_{j}(\mathcal{M}, \mathcal{N})\right) e^{-s_{j}(\mathcal{M}, \mathcal{N})}
\end{array}\right] \\
& =\mathcal{D} v(\mathcal{K}, \mathcal{N})+\mathcal{D} v(\mathcal{M}, \mathcal{N})
\end{aligned}
$$

Box IV

Proposition 3.4. For CIFSs $\mathcal{K}, \mathcal{M}$, and $\mathcal{N}$ defined on $\mathcal{X}$, we have:

(i) $\mathcal{D} v(\mathcal{K}, \mathcal{N})+\mathcal{D} v(\mathcal{M}, \mathcal{N})-\mathcal{D} v(\mathcal{K} \cup \mathcal{M}, \mathcal{N}) \geq 0$

(ii) $\mathcal{D} v(\mathcal{K}, \mathcal{N})+\mathcal{D} v(\mathcal{M}, \mathcal{N})-\mathcal{D} v(\mathcal{K} \cap \mathcal{M}, \mathcal{N}) \geq 0$.

Proof. Based on Proposition 3.2, we have $\mathcal{D} v(\mathcal{K}, \mathcal{N})+$
$\mathcal{D} v(\mathcal{M}, \mathcal{N})-\mathcal{D} v(\mathcal{K} \cup \mathcal{M}, \mathcal{N})=\mathcal{D} v(\mathcal{K} \cap \mathcal{M}, \mathcal{N})$ and based on Theorem 3.1, we have $\mathcal{D} v(\mathcal{K} \cap \mathcal{M}, \mathcal{N}) \geq 0$. Therefore, $\mathcal{D} v(\mathcal{K}, \mathcal{N})+\mathcal{D} v(\mathcal{M}, \mathcal{N})-\mathcal{D} v(\mathcal{K} \cup \mathcal{M}, \mathcal{N}) \geq$ 0 . $\square$

Proposition 3.5. For CIFSs $\mathcal{K}$ and $\mathcal{M}$ defined on $\mathcal{X}$, we have: 
(i) $\mathcal{D} v(\mathcal{K}, \mathcal{M})=\mathcal{D} v\left(\mathcal{K}^{c}, \mathcal{M}^{c}\right)$;

(ii) $\mathcal{D} v\left(\mathcal{K}^{c}, \mathcal{M}\right)=\mathcal{D} v\left(\mathcal{K}, \mathcal{M}^{c}\right)$;

(iii) $\mathcal{D} v(\mathcal{K}, \mathcal{M})+\mathcal{D} v\left(\mathcal{K}^{c}, \mathcal{M}\right)=\mathcal{D} v\left(\mathcal{K}^{c}, \mathcal{M}^{c}\right)+\mathcal{D} v(\mathcal{K}$, $\left.\mathcal{M}^{c}\right)$.

Proof. They can be directly proved through Definition 3.3.

Next, we define the weighted exponential divergence measure between CIFSs. In this respect, let $\kappa_{j}>$ 0 be the weight vector of $x_{j} \in \mathcal{X}$ with $\sum_{j=1}^{n} \kappa_{j}=1$.

Definition 3.4. A weighted exponential divergence measure between two CIFSs $\mathcal{K}$ and $\mathcal{M}$ is defined as:

$$
\begin{gathered}
\mathcal{D} v_{\kappa}(\mathcal{K}, \mathcal{M})=\frac{1}{4\left(1-e^{-1}\right)} \sum_{j=1}^{n} \kappa_{j} \\
\left(\begin{array}{c}
4-\left(1-t_{j}(\mathcal{K}, \mathcal{M})\right) \exp \left(t_{j}(\mathcal{K}, \mathcal{M})\right) \\
-\left(1+t_{j}(\mathcal{K}, \mathcal{M})\right) \exp \left(-t_{j}(\mathcal{K}, \mathcal{M})\right) \\
-\left(1-s_{j}(\mathcal{K}, \mathcal{M})\right) \exp \left(s_{j}(\mathcal{K}, \mathcal{M})\right) \\
-\left(1+s_{j}(\mathcal{K}, \mathcal{M})\right) \exp \left(-s_{j}(\mathcal{K}, \mathcal{M})\right)
\end{array}\right),
\end{gathered}
$$

where:

$t_{j}(\mathcal{K}, \mathcal{M})=\frac{\left(r_{\mathcal{K}}\left(x_{j}\right)-r_{\mathcal{M}}\left(x_{j}\right)\right)-\left(k_{\mathcal{K}}\left(x_{j}\right)-k_{\mathcal{M}}\left(x_{j}\right)\right)}{2}$,

and:

$s_{j}(\mathcal{K}, \mathcal{M})$

$$
=\frac{\left(w_{r_{\mathcal{K}}}\left(x_{j}\right)-w_{r_{\mathcal{M}}}\left(x_{j}\right)\right)-\left(w_{k_{\mathcal{K}}}\left(x_{j}\right)-w_{k_{\mathcal{M}}}\left(x_{j}\right)\right)}{2} .
$$

If $\kappa=(1 / n, 1 / n, \cdots, 1 / n)^{T}$, Eq. (11) becomes Eq. (10). Further, the measure defined in Eq. (11) also satisfies the axioms of DvMs, thus $0 \leq \mathcal{D} v_{\kappa}(\mathcal{K}, \mathcal{M}) \leq 1$.

Proposition 3.6. Let $\mathcal{K}, \mathcal{M}$, and $\mathcal{N}$ be three CIFSs defined on $\mathcal{X}=\left\{x_{1}, x_{2}, \cdots, x_{n}\right\}$ such that for every $x_{j} \in \mathcal{X}$ either $\mathcal{K}\left(x_{j}\right) \subseteq \mathcal{M}\left(x_{j}\right)$ or $\mathcal{M}\left(x_{j}\right) \subseteq \mathcal{K}\left(x_{j}\right)$. Then:

(i) $\mathcal{D} v_{\kappa}(\mathcal{K} \cup \mathcal{M}, \mathcal{K} \cap \mathcal{M})=\mathcal{D} v_{\kappa}(\mathcal{K}, \mathcal{M})$;

(ii) $\mathcal{D} v_{\kappa}(\mathcal{K} \cap \mathcal{M}, \mathcal{K} \cup \mathcal{M})=\mathcal{D} v_{\kappa}(\mathcal{K}, \mathcal{M})$;

(iii) $\mathcal{D} v_{\kappa}(\mathcal{K} \cup \mathcal{M}, \mathcal{N})+\mathcal{D} v_{\kappa}(\mathcal{K} \cap \mathcal{M}, \mathcal{N})=\mathcal{D} v_{\kappa}(\mathcal{K}$, $\mathcal{N})+\mathcal{D} v_{\kappa}(\mathcal{M}, \mathcal{N})$

(iv) $\mathcal{D} v_{\kappa}(\mathcal{K}, \mathcal{K} \cup \mathcal{M})+\mathcal{D} v_{\kappa}(\mathcal{K}, \mathcal{K} \cap \mathcal{M})=\mathcal{D} v_{\kappa}(\mathcal{K}, \mathcal{M})$;

(v) $\mathcal{D} v_{\kappa}(\mathcal{M}, \mathcal{K} \cup \mathcal{M})+\mathcal{D} v_{\kappa}(\mathcal{M}, \mathcal{K} \cap \mathcal{M})=\mathcal{D} v_{\kappa}(\mathcal{K}$, $\mathcal{M})$

(vi) $\mathcal{D} v_{\kappa}(\mathcal{K}, \mathcal{N})+\mathcal{D} v_{\kappa}(\mathcal{M}, \mathcal{N})-\mathcal{D} v_{\kappa}(\mathcal{K} \cup \mathcal{M}, \mathcal{N}) \geq 0$;

(vii) $\mathcal{D} v_{\kappa}(\mathcal{K}, \mathcal{N})+\mathcal{D} v_{\kappa}(\mathcal{M}, \mathcal{N})-\mathcal{D} v_{\kappa}(\mathcal{K} \cap \mathcal{M}, \mathcal{N}) \geq 0$;

(viii) $\mathcal{D} v_{\kappa}(\mathcal{K}, \mathcal{M})=\mathcal{D} v_{\kappa}\left(\mathcal{K}^{c}, \mathcal{M}^{c}\right)$; (ix) $\mathcal{D} v_{\kappa}\left(\mathcal{K}^{c}, \mathcal{M}\right)=\mathcal{D} v_{\kappa}\left(\mathcal{K}, \mathcal{M}^{c}\right)$;

(x) $\mathcal{D} v_{\kappa}(\mathcal{K}, \mathcal{M})+\mathcal{D} v_{\kappa}\left(\mathcal{K}^{c}, \mathcal{M}\right)=\mathcal{D} v_{\kappa}\left(\mathcal{K}^{c}, \mathcal{M}^{c}\right)+$ $\mathcal{D} v_{\kappa}\left(\mathcal{K}, \mathcal{M}^{c}\right)$.

Proof. These are similar to the proofs of Propositions 3.1-3.5.

\section{The proposed approach based on DM}

Assume that there is a set of alternatives $\mathcal{A}_{1}, \mathcal{A}_{2}, \cdots, \mathcal{A}_{m}$ under " $n$ " criteria $\mathcal{C}_{1}, \mathcal{C}_{2}, \cdots, \mathcal{C}_{n}$. Let $\kappa_{q}>0$ be the weight vector corresponding to the criterion $\mathcal{C}_{q}$ with $\sum_{q=1}^{n} \kappa_{q}=1$. An expert evaluated these different alternatives under the set of criteria and gave their preferences in terms of CIFNs $\alpha_{p q}$. The collective information of such rating is represented as a matrix $\mathcal{D}=\left(\alpha_{p q}\right)_{m \times n}$.

Yingming [53] recommended an approach to determining the criteria weights more subjectively and called it a method for maximizing deviations. In this method, the weight vector $\kappa$ should be chosen such that deviations of all criteria corresponding to alternatives become maximum. For an arbitrary criterion $C_{q}(q=$ $1,2, \cdots, n)$, the deviation of alternative $\mathcal{A}_{p}$ to other alternatives is given as:

$$
\mathscr{D}_{p q}\left(\kappa_{q}\right)=\sum_{u=1}^{m} \mathcal{D} v\left(\alpha_{p q}, \alpha_{u q}\right) \kappa_{q} .
$$

Then, total deviation of the criterion $C_{q}$ from all the alternatives is given as:

$$
\mathscr{D}_{q}=\sum_{p=1}^{m} \sum_{u=1}^{m} \mathcal{D} v\left(\alpha_{p q}, \alpha_{u q}\right) \kappa_{q} .
$$

Further, the deviations of all criteria from all alternatives are obtained as:

$$
\mathscr{D}=\sum_{q=1}^{n} \sum_{p=1}^{m} \sum_{u=1}^{m} \mathcal{D} v\left(\alpha_{p q}, \alpha_{u q}\right) \kappa_{q} .
$$

Let $\triangle$ be the set of weight information known in any of the following forms:

Form 1. A weak ranking: $\kappa_{i} \geq \kappa_{j}$;

Form 2. A strict ranking: $\kappa_{i}-\kappa_{j} \geq \sigma_{i},\left(\sigma_{i}>0\right)$;

Form 3. A ranking with multiples: $\kappa_{i} \geq \sigma_{i} \kappa_{j},(0 \leq$ $\left.\sigma_{i} \leq 1\right)$;

Form 4. An interval form: $\lambda_{i} \leq \kappa_{i} \leq \lambda_{i}+\delta_{i}$, $(0 \leq$ $\left.\lambda_{i} \leq \lambda_{i}+\delta_{i} \leq 1\right)$;

Form 5. A ranking of differences: $\kappa_{i}-\kappa_{j} \geq \kappa_{k}-\kappa_{l}$, $(j \neq k \neq l)$.

Now, based on $\mathscr{D}$, the nonlinear optimization model can be constructed to find the optimal weights of the criteria by assuming that the information related to 
attribute weights is partially known, as shown in the following:

$$
\max \quad \mathscr{D}=\sum_{q=1}^{n} \sum_{p=1}^{m} \sum_{u=1}^{m} \mathcal{D} v\left(\alpha_{p q}, \alpha_{u q}\right) \kappa_{q},
$$

subject to: $\kappa_{q} \in \triangle, \quad \sum_{q=1}^{n} \kappa_{q}=1, \quad \kappa_{q}>0$,

where $\mathcal{D} v\left(\alpha_{p q}, \alpha_{u q}\right)$ is determined using Eq. (10).

In case the information about the attribute weights is completely unknown, another nonlinear optimization model can be established as follows:

$$
\begin{aligned}
& \max \quad \mathscr{D}=\sum_{q=1}^{n} \sum_{p=1}^{m} \sum_{u=1}^{m} \mathcal{D} v\left(\alpha_{p q}, \alpha_{u q}\right) \kappa_{q}, \\
& \text { subject to: } \quad \sum_{q=1}^{n} \kappa_{q}^{2}=1, \quad \kappa_{q}>0 .
\end{aligned}
$$

In order to obtain the solution of the problem mentioned in Eq. (13), consider a function:

$g\left(\kappa_{q}, \lambda\right)=\sum_{q=1}^{n} \sum_{p=1}^{m} \sum_{u=1}^{m} \mathcal{D} v\left(\alpha_{p q}, \alpha_{u q}\right) \kappa_{q}+\lambda\left(\sum_{q=1}^{n} \kappa_{q}^{2}-1\right)$

where $\lambda$ is Lagrange's multiplier. Now:

$$
\begin{aligned}
& \frac{\partial g}{\partial \kappa_{q}}=\sum_{p=1}^{m} \sum_{u=1}^{m} \mathcal{D} v\left(\alpha_{p q}, \alpha_{u q}\right)+\lambda, \\
& \frac{\partial g}{\partial \lambda}=\sum_{q=1}^{n} \kappa_{q}^{2}-1 .
\end{aligned}
$$

Now, upon setting Eqs. (15) and (16) equal to zero and then, solving them, we obtain:

$$
\kappa_{q}=\frac{\sum_{p=1}^{m} \sum_{u=1}^{m} \mathcal{D} v\left(\alpha_{p q}, \alpha_{u q}\right)}{\sqrt{\sum_{q=1}^{n}\left(\sum_{p=1}^{m} \sum_{u=1}^{m} \mathcal{D} v\left(\alpha_{p q}, \alpha_{u q}\right)\right)^{2}}} .
$$

Further, the normalized value of $\kappa_{q}$ can be obtained as follows:

$$
\kappa_{q}^{*}=\frac{\kappa_{q}}{\sum_{q=1}^{n} \kappa_{q}}=\frac{\sum_{p=1}^{m} \sum_{u=1}^{m} \mathcal{D} v\left(\alpha_{p q}, \alpha_{u q}\right)}{\sum_{q=1}^{n} \sum_{p=1}^{m} \sum_{u=1}^{m} \mathcal{D} v\left(\alpha_{p q}, \alpha_{u q}\right)} .
$$

These models should be solved to obtain the optimal weights $\kappa=\left(\kappa_{1}, \kappa_{2}, \cdots, \kappa_{n}\right)^{T}$.

Based on the collective information and the weight vector $\kappa$, the following steps are proposed to compute the finest alternatives from the given ones:

Step 1: The information about the alternatives is represented as a decision matrix $\mathcal{D}$, as shown in the following:

$$
\mathcal{D}=\begin{gathered}
\mathcal{C}_{1} \\
\mathcal{A}_{1} \\
\mathcal{A}_{2} \\
\vdots \\
\mathcal{A}_{m}
\end{gathered}\left(\begin{array}{cccc}
\alpha_{11} & \alpha_{12} & \ldots & \mathcal{C}_{n} \\
\alpha_{21} & \alpha_{22} & \ldots & \alpha_{1 n} \\
\vdots & \vdots & \ddots & \alpha_{2 n} \\
\alpha_{m 1} & \alpha_{m 2} & \ldots & \alpha_{m n}
\end{array}\right) .
$$

Step 2: Normalize the information, if required; then, the obtained matrix $\mathcal{R}=\left(\zeta_{p q}\right)$ where $\zeta_{p q}$ is obtained through Eq. (20):

$$
\zeta_{p q}=\left\{\begin{array}{l}
\text { For benefit type criteria : } \\
\left(\left(r_{p q}, w_{r_{p q}}\right),\left(k_{p q}, w_{k_{p q}}\right)\right) ; \\
\text { For cost type criteria : } \\
\left(\left(k_{p q}, w_{k_{p q}}\right),\left(r_{p q}, w_{r_{p q}}\right)\right) ;
\end{array}\right.
$$

Step 3: Formulate the optimization model using either Eq. (12) or Eq. (18) according to the known information of the weight vector and solve it.

Step 4: Construct the ideal alternative denoted by $\mathcal{A}^{*}$ as:

$$
\mathcal{A}^{*}=\left\{\left(\mathcal{C}_{q},\left(r_{q}, w_{r_{q}}\right),\left(k_{q}, w_{k_{q}}\right)\right) \mid q=1,2, \cdots, n\right\},
$$

where $r_{q}=\max _{p}\left\{r_{p q}\right\} ; k_{q}=\min _{p}\left\{k_{p q}\right\} ; w_{r_{q}}=$ $\max _{p}\left\{w_{r_{p q}}\right\}$ and $w_{k_{q}}=\min _{p}\left\{w_{k_{p q}}\right\} \forall q=1,2, \cdots, n$.

Step 5: Compute the DvM for the alternative $\mathcal{A}_{p}(p=1,2, \cdots, m)$ from $\mathcal{A}^{*}$ as:

$$
\begin{aligned}
\mathcal{D} v_{\kappa}\left(\mathcal{A}_{p}, \mathcal{A}^{*}\right) & =\frac{1}{4\left(1-e^{-1}\right)} \sum_{q=1}^{n} \kappa_{q} \\
& \left(\begin{array}{c}
4-\left(1-t_{q}\left(\mathcal{A}_{p}, \mathcal{A}^{*}\right)\right) \exp \left(t_{q}\left(\mathcal{A}_{p}, \mathcal{A}^{*}\right)\right) \\
-\left(1+t_{q}\left(\mathcal{A}_{p}, \mathcal{A}^{*}\right)\right) \exp \left(-t_{q}\left(\mathcal{A}_{p}, \mathcal{A}^{*}\right)\right) \\
-\left(1-s_{q}\left(\mathcal{A}_{p}, \mathcal{A}^{*}\right)\right) \exp \left(s_{q}\left(\mathcal{A}_{p}, \mathcal{A}^{*}\right)\right) \\
-\left(1+s_{q}\left(\mathcal{A}_{p}, \mathcal{A}^{*}\right)\right) \exp \left(-s_{q}\left(\mathcal{A}_{p}, \mathcal{A}^{*}\right)\right)
\end{array}\right) .
\end{aligned}
$$

Step 6: Based on the argument of $\mathcal{D} v_{\kappa}$ given as $\arg \min _{1 \leq p \leq m}\left\{\mathcal{D} v_{\kappa}\left(\mathcal{A}_{p}, \mathcal{A}^{*}\right)\right\}$, order the alternatives and select the desired one.

\section{Illustrative example}

To illustrate the approach, a case study was taken into account concerning the entrepreneur to purchase a new machine out of four different models denoted by $\mathcal{A}_{1}$, $\mathcal{A}_{2}, \mathcal{A}_{3}$, and $\mathcal{A}_{4}$ with different dates of production of each model. The accessibility of such machines was measured under four different criteria:

$$
\begin{aligned}
& \mathcal{C}_{1} \text { : "Reliability", } \\
& \mathcal{C}_{2} \text { : "Safety", } \\
& \mathcal{C}_{3} \text { : "Cost", }
\end{aligned}
$$


$\mathcal{C}_{4}$ : "Productivity for selecting machine".

These factors change with the change of the production dates. To evaluate each machine under such factors, preferences are taken from the expert in CIFNs. The steps of the proposed method are illustrated in the following:

Step 1: The given information is collected in terms of CIFNs, which is summarized in Table 2. In this matrix, the entry corresponding to the machine $\mathcal{A}_{1}$ indicates that an expert during the evaluation agrees that it is reliable up to $70 \%$ under $\mathcal{C}_{1}$ and unreliable at most $10 \%$. Similarly, concerning the production date, he feels that $50 \%$ is compatible and $30 \%$ is incompatible with $\mathcal{C}_{1}$. In a similar manner, all data of matrix $\mathcal{D}$ can be interpreted;

Step 2: As $\mathcal{C}_{3}$ is the cost type, through Eq. (20), the normalized data are presented in Table 3;

Step 3: If we initially assume that the partial information about the weight vector corresponding to the criteria is partially known given as $\triangle=$ $\left\{0.2 \leq \kappa_{1} \leq 0.4,0.15 \leq \kappa_{2} \leq 0.25,0.25 \leq \kappa_{3} \leq\right.$ $\left.0.3,0.1 \leq \kappa_{4} \leq 0.25\right\}$ such that $\kappa_{1}+\kappa_{2}+\kappa_{3}+\kappa_{4}=1$. Then, through Eq. (12), an optimization model can be formulated as:

$$
\begin{aligned}
\max \quad \mathscr{D}(\kappa)= & 0.1610 \kappa_{1}+0.0890 \kappa_{2} \\
& +0.1272 \kappa_{3}+0.0711 \kappa_{4},
\end{aligned}
$$

subject to: $\quad 0.20 \leq \kappa_{1} \leq 0.40$,

$$
\begin{aligned}
& 0.15 \leq \kappa_{2} \leq 0.25, \\
& 0.25 \leq \kappa_{3} \leq 0.30, \\
& 0.10 \leq \kappa_{4} \leq 0.25,
\end{aligned}
$$

$$
\sum_{q=1}^{4} \kappa_{q}=1, \quad \kappa_{q}>0
$$

After solving, we get $\kappa=(0.4,0.2,0.3,0.1)^{T}$. On the contrary, if we assume that the information related to the criteria weights is completely unknown, the weight vector can be obtained as $\kappa=$ $(0.3592,0.1985,0.2838,0.1585)^{T}$ using Eq. (18).

Step 4: Based on the matrix $\mathcal{R}$, the ideal alternative is taken as:

$$
\mathcal{A}^{*}=\left\{\begin{array}{l}
\left(\mathcal{C}_{1},(0.7,0.8),(0.1,0.1)\right) \\
\left(\mathcal{C}_{2},(0.7,0.9),(0.2,0.1)\right) \\
\left(\mathcal{C}_{3},(0.7,0.7),(0.1,0.1)\right) \\
\left(\mathcal{C}_{4},(0.7,0.7),(0.1,0.1)\right)
\end{array}\right\}
$$

Step 5: With $\kappa=(0.4,0.2,0.3,0.1)^{T}$, the exponential divergence measure values are obtained by Eq. (21) and we have:

$$
\begin{array}{ll}
\mathcal{D} v_{\kappa}\left(\mathcal{A}_{1}, \mathcal{A}^{*}\right)=0.0276, & \mathcal{D} v_{\kappa}\left(\mathcal{A}_{2}, \mathcal{A}^{*}\right)=0.0139, \\
\mathcal{D} v_{\kappa}\left(\mathcal{A}_{3}, \mathcal{A}^{*}\right)=0.1012, & \mathcal{D} v_{\kappa}\left(\mathcal{A}_{4}, \mathcal{A}^{*}\right)=0.0425 .
\end{array}
$$

In case of using $\kappa=(0.3592,0.1985,0.2838,0.1585)^{T}$, the measurement values are obtained as:

$$
\begin{array}{ll}
\mathcal{D} v_{\kappa}\left(\mathcal{A}_{1}, \mathcal{A}^{*}\right)=0.0281, & \mathcal{D} v_{\kappa}\left(\mathcal{A}_{2}, \mathcal{A}^{*}\right)=0.0145, \\
\mathcal{D} v_{\kappa}\left(\mathcal{A}_{3}, \mathcal{A}^{*}\right)=0.0936, & \mathcal{D} v_{\kappa}\left(\mathcal{A}_{4}, \mathcal{A}^{*}\right)=0.0425 .
\end{array}
$$

Step 6: Through these values, the ordering of the alternative will be $\mathcal{A}_{2} \succ \mathcal{A}_{1} \succ \mathcal{A}_{4} \succ \mathcal{A}_{3}$ where " $\succ$ " refers "preferred to". Hence, $\mathcal{A}_{2}$ is the finest one.

Table 2. Input data for the problem in Complex Intuitionistic Fuzzy Number (CIFN) format.

\begin{tabular}{ccccc}
\hline & $\mathcal{C}_{\mathbf{1}}$ & $\mathcal{C}_{\mathbf{2}}$ & $\mathcal{C}_{\mathbf{3}}$ & $\mathcal{C}_{\boldsymbol{4}}$ \\
\hline $\mathcal{A}_{\mathbf{1}}$ & $((0.7,0.5),(0.1,0.3))$ & $((0.4,0.5),(0.3,0.4))$ & $((0.2,0.1),(0.6,0.6))$ & $((0.5,0.4),(0.1,0.3))$ \\
$\mathcal{A}_{\mathbf{2}}$ & $((0.7,0.6),(0.3,0.3))$ & $((0.4,0.9),(0.2,0.1))$ & $((0.2,0.3),(0.7,0.7))$ & $((0.4,0.6),(0.3,0.1))$ \\
$\mathcal{A}_{\mathbf{3}}$ & $((0.3,0.4),(0.6,0.4))$ & $((0.6,0.6),(0.3,0.4))$ & $((0.5,0.6),(0.3,0.4))$ & $((0.7,0.7),(0.1,0.1))$ \\
$\mathcal{A}_{\boldsymbol{4}}$ & $((0.4,0.8),(0.5,0.1))$ & $((0.7,0.3),(0.3,0.3))$ & $((0.1,0.3),(0.6,0.5))$ & $((0.5,0.5),(0.3,0.4))$ \\
\hline
\end{tabular}

Table 3. Normalized information data.

\begin{tabular}{ccccc}
\hline & $\mathcal{C}_{\boldsymbol{1}}$ & $\mathcal{C}_{\mathbf{2}}$ & $\mathcal{C}_{\mathbf{3}}$ & $\mathcal{C}_{\mathbf{4}}$ \\
\hline $\mathcal{A}_{\mathbf{1}}$ & $((0.7,0.5),(0.1,0.3))$ & $((0.4,0.5),(0.3,0.4))$ & $((0.6,0.6),(0.2,0.1))$ & $((0.5,0.4),(0.1,0.3))$ \\
$\mathcal{A}_{\mathbf{2}}$ & $((0.7,0.6),(0.3,0.3))$ & $((0.4,0.9),(0.2,0.1))$ & $((0.7,0.7),(0.2,0.3))$ & $((0.4,0.6),(0.3,0.1))$ \\
$\mathcal{A}_{\mathbf{3}}$ & $((0.3,0.4),(0.6,0.4))$ & $((0.6,0.6),(0.3,0.4))$ & $((0.3,0.4),(0.5,0.6))$ & $((0.7,0.7),(0.1,0.1))$ \\
$\mathcal{A}_{\boldsymbol{4}}$ & $((0.4,0.8),(0.5,0.1))$ & $((0.7,0.3),(0.3,0.3))$ & $((0.6,0.5),(0.1,0.3))$ & $((0.5,0.5),(0.3,0.4))$ \\
\hline
\end{tabular}


Table 4. Comparative study results under Intuitionistic Fuzzy Set (IFS) environment.

\begin{tabular}{llcccccc}
\hline \multirow{2}{*}{ Ref. } & \multirow{2}{*}{ Method based on } & \multicolumn{4}{c}{ Measurement values of } & \multirow{2}{*}{ Ranking } \\
\cline { 3 - 6 } & & $\mathcal{A}_{\mathbf{1}}$ & $\mathcal{A}_{\mathbf{2}}$ & $\mathcal{A}_{\mathbf{3}}$ & $\mathcal{A}_{4}$ & \\
\hline Maheshwari and Srivastava [33] & Divergence measure & 0.0161 & 0.0491 & 0.0515 & 0.0371 & $\mathcal{A}_{1} \succ \mathcal{A}_{4} \succ \mathcal{A}_{2} \succ \mathcal{A}_{3}$ \\
Ohlan [30] & Divergence measure & 0.0605 & 0.0986 & 0.3893 & 0.1717 & $\mathcal{A}_{1} \succ \mathcal{A}_{2} \succ \mathcal{A}_{4} \succ \mathcal{A}_{3}$ \\
Srivastava and Maheshwari [34] & Divergence measure & 0.1125 & 0.1722 & 0.1926 & 0.1520 & $\mathcal{A}_{1} \succ \mathcal{A}_{4} \succ \mathcal{A}_{2} \succ \mathcal{A}_{3}$ \\
Garg et al. [31] & Divergence measure & 0.0563 & 0.2105 & 0.1697 & 0.1606 & $\mathcal{A}_{1} \succ \mathcal{A}_{4} \succ \mathcal{A}_{3} \succ \mathcal{A}_{2}$ \\
Shen et al. [35] & Distance measure & 0.0857 & 0.1439 & 0.3644 & 0.2102 & $\mathcal{A}_{1} \succ \mathcal{A}_{2} \succ \mathcal{A}_{4} \succ \mathcal{A}_{3}$ \\
Ye [36] & Similarity measure & 0.9816 & 0.9500 & 0.8231 & 0.9126 & $\mathcal{A}_{1} \succ \mathcal{A}_{2} \succ \mathcal{A}_{4} \succ \mathcal{A}_{3}$ \\
Song et al. [37] & Similarity measure & 0.9888 & 0.9637 & 0.9155 & 0.9573 & $\mathcal{A}_{1} \succ \mathcal{A}_{2} \succ \mathcal{A}_{4} \succ \mathcal{A}_{3}$ \\
\hline
\end{tabular}

\subsection{Comparative analysis with CIFS studies}

To check the consistency of the method with some existing studies $[42,44,45,52]$ under the CIFS environment, an analysis is conducted by their method and the corresponding results are discussed below:

(i) By applying the method proposed by Alkouri and Salleh [42] based on the distance measure ' $d_{1}$ ' to the given information, we get $d_{1}\left(\mathcal{A}_{1}, \mathcal{A}^{*}\right)=0.1500$, $d_{1}\left(\mathcal{A}_{2}, \mathcal{A}^{*}\right)=0.1325, d_{1}\left(\mathcal{A}_{3}, \mathcal{A}^{*}\right)=0.3310$, and $d_{1}\left(\mathcal{A}_{4}, \mathcal{A}^{*}\right)=0.1885$. Thus, ordering is $\mathcal{A}_{2} \succ \mathcal{A}_{1} \succ$ $\mathcal{A}_{4} \succ \mathcal{A}_{3}$ and $\mathcal{A}_{2}$ is the best alternative;

(ii) By applying the method of Rani and Garg [44] based on Hamming distance measure ' $d_{2}$ ', we get the values as $d_{2}\left(\mathcal{A}_{1}, \mathcal{A}^{*}\right)=0.1450, d_{2}\left(\mathcal{A}_{2}, \mathcal{A}^{*}\right)=$ $0.1125, d_{2}\left(\mathcal{A}_{3}, \mathcal{A}^{*}\right)=0.3200$, and $d_{2}\left(\mathcal{A}_{4}, \mathcal{A}^{*}\right)=$ 0.1725 . Hence, it is seen that $\mathcal{A}_{2}$ is again the best alternative;

(iii) By applying the method of Garg and Rani [45] based on correlation coefficient ' $C$ ', we obtain the indices' values as $C\left(\mathcal{A}_{1}, \mathcal{A}^{*}\right)=0.9407$, $C\left(\mathcal{A}_{2}, \mathcal{A}^{*}\right)=0.9571, C\left(\mathcal{A}_{3}, \mathcal{A}^{*}\right)=0.7547$, and $C\left(\mathcal{A}_{4}, \mathcal{A}^{*}\right)=0.8926$. Clearly, it is seen that the best alternative is $\mathcal{A}_{2}$;

(iv) By performing the similarity measure $S_{1}$, as proposed by Garg and Rani [52], on the considered information under the CIFS environment, we get the measurement value of each alternative as $S_{1}\left(\mathcal{A}_{1}, \mathcal{A}^{*}\right)=0.6733, S_{1}\left(\mathcal{A}_{2}, \mathcal{A}^{*}\right)=0.7663$, $S_{1}\left(\mathcal{A}_{3}, \mathcal{A}^{*}\right)=0.5930$, and $S_{1}\left(\mathcal{A}_{4}, \mathcal{A}^{*}\right)=0.6378$. Thus, from it, we conclude that the best alternative is $\mathcal{A}_{2}$.

From it, we conclude that their position of the given alternatives coincides with the given ones, which validates the feasibility of the method.

\subsection{Comparative analysis with IFS studies}

Given that IFS is one of the special cases of CIFS with zero phase terms in each CIFNs, in order to check their performance under the IFS environment, we conduct a comparative study with several existing approaches $[30,31,33-37]$. The results are obtained and listed in Table 4 and it is determined that $\mathcal{A}_{1}$ is the best alternative. However, from the proposed approach, it can be concluded that $\mathcal{A}_{2}$ is the best alternative. This change in the optimal ranking order is quite significant. This is due to the consideration of the computational procedure in the considered environment. For example, in [30,31,33-37] approaches, only one grade of MDs and NMDs is taken into account. In relation to the considered problem, it can be claimed that a great focus is devoted to the model of the machine in a condition where the production date of each model is neglected. Thus, based on the analysis, $\mathcal{A}_{1}$ is the best machine when there are no limits on the production date. However, in the proposed work, we have investigated the theory based on both the model as well as production dates simultaneously and, hence, it is concluded that machine $\mathcal{A}_{2}$ is the best with the production date.

\subsection{Verification and comparative analysis}

To generalize the capability of CIFS with respect to the features of IFS, some examples are given in the following:

Example 5.1. Consider a DMP which consists of five alternatives in the form of regions, namely:

$$
\begin{aligned}
& \mathcal{A}_{1}: \text { Lalitpur, } \\
& \mathcal{A}_{2}: \text { Kathmandu, } \\
& \mathcal{A}_{3}: \text { Gorkha, } \\
& \mathcal{A}_{4}: \text { Bhaktapur } \\
& \mathcal{A}_{5}: \text { Makwanpur }
\end{aligned}
$$

which are affected by the earthquakes, racked Nepal on 25 April 2015. The given task is to identify the most damaged region so that necessary facilities including $\mathcal{C}_{1}$ (food), $\mathcal{C}_{2}$ (shelter), $\mathcal{C}_{3}$ (clothes), and $\mathcal{C}_{4}$ (medical requirements) are provided to victims. Let $\kappa=(0.30,0.25,0.15,0.30)^{T}$ be the priority weight of them. Before allocating, an expert evaluates the given 
Table 5. Input preference for Example 5.1.

\begin{tabular}{ccccc}
\hline & $\mathcal{C}_{1}$ & $\mathcal{C}_{2}$ & $\mathcal{C}_{3}$ & $\mathcal{C}_{4}$ \\
\hline $\mathcal{A}_{\mathbf{1}}$ & $((0.6,0.7),(0.1,0.2))$ & $((0.9,0.8),(0.1,0.1))$ & $((0.5,0.4),(0.3,0.4))$ & $((0.6,0.4),(0.2,0.1))$ \\
$\mathcal{A}_{\mathbf{2}}$ & $((0.4,0.2),(0.3,0.1))$ & $((0.5,0.3),(0.1,0.1))$ & $((0.6,0.4),(0.2,0.3))$ & $((0.8,0.6),(0.1,0.2))$ \\
$\mathcal{A}_{\mathbf{3}}$ & $((0.7,0.7),(0.1,0.2))$ & $((0.4,0.6),(0.3,0.1))$ & $((0.7,0.7),(0.1,0.1))$ & $((0.6,0.5),(0.3,0.4))$ \\
$\mathcal{A}_{4}$ & $((0.7,0.6),(0.3,0.3))$ & $((0.4,0.9),(0.2,0.1))$ & $((0.7,0.7),(0.2,0.3))$ & $((0.5,0.3),(0.3,0.6))$ \\
$\mathcal{A}_{\mathbf{5}}$ & $((0.2,0.8),(0.5,0.1))$ & $((0.7,0.3),(0.3,0.3))$ & $((0.6,0.5),(0.1,0.3))$ & $((0.6,0.5),(0.3,0.4))$ \\
\hline
\end{tabular}

Table 6. Comparative analysis of Example 5.1 with existing studies.

\begin{tabular}{|c|c|c|c|c|c|c|c|}
\hline \multicolumn{8}{|c|}{ Study under CIFS environment } \\
\hline \multicolumn{2}{|c|}{ Approach } & \multicolumn{5}{|c|}{ Measurement value of $\mathcal{B}$ from } & \multirow{2}{*}{ Ranking order } \\
\hline Ref. & Method based on & $\mathcal{A}_{1}$ & $\mathcal{A}_{2}$ & $\mathcal{A}_{3}$ & $\mathcal{A}_{4}$ & $\mathcal{A}_{5}$ & \\
\hline Alkouri and Salleh [42] & Distance measure & 0.1817 & 0.1917 & 0.1400 & 0.2167 & 0.2600 & $\mathcal{A}_{3} \succ \mathcal{A}_{1} \succ \mathcal{A}_{2} \succ \mathcal{A}_{4} \succ \mathcal{A}_{5}$ \\
\hline Rani and Garg [44] & Euclidean distance measure & 0.1871 & 0.1803 & 0.1374 & 0.2086 & 0.2225 & $\mathcal{A}_{3} \succ \mathcal{A}_{2} \succ \mathcal{A}_{1} \succ \mathcal{A}_{4} \succ \mathcal{A}_{5}$ \\
\hline Garg and Rani [45] & Correlation measure & 0.8965 & 0.9087 & 0.9439 & 0.8747 & 0.8351 & $\mathcal{A}_{3} \succ \mathcal{A}_{2} \succ \mathcal{A}_{1} \succ \mathcal{A}_{4} \succ \mathcal{A}_{5}$ \\
\hline Garg and Rani [52] & Similarity measure & 0.6912 & 0.6231 & 0.7400 & 0.6501 & 0.5762 & $\mathcal{A}_{3} \succ \mathcal{A}_{2} \succ \mathcal{A}_{1} \succ \mathcal{A}_{4} \succ \mathcal{A}_{5}$ \\
\hline \multirow[t]{2}{*}{ Proposed method } & Divergence measure & 0.0325 & 0.0185 & 0.0171 & 0.0391 & 0.0552 & $\mathcal{A}_{3} \succ \mathcal{A}_{2} \succ \mathcal{A}_{1} \succ \mathcal{A}_{4} \succ \mathcal{A}_{5}$ \\
\hline & & \multicolumn{5}{|c|}{ Study under IFS environment } & \\
\hline \multicolumn{2}{|c|}{ Approach } & \multicolumn{5}{|c|}{ Measurement value of $\mathcal{B}$ from } & \multirow{2}{*}{ Ranking order } \\
\hline Ref. & Method based on & $\mathcal{A}_{1}$ & $\mathcal{A}_{2}$ & $\mathcal{A}_{3}$ & $\mathcal{A}_{4}$ & $\mathcal{A}_{5}$ & \\
\hline Zeng and Li [17] & Correlation coefficient & 0.8740 & 0.8874 & 0.9442 & 0.8822 & 0.8262 & $\mathcal{A}_{3} \succ \mathcal{A}_{2} \succ \mathcal{A}_{4} \succ \mathcal{A}_{1} \succ \mathcal{A}_{5}$ \\
\hline $\mathrm{Ye}[18]$ & Correlation coefficient & 0.8808 & 0.9106 & 0.9551 & 0.9246 & 0.8250 & $\mathcal{A}_{3} \succ \mathcal{A}_{4} \succ \mathcal{A}_{2} \succ \mathcal{A}_{1} \succ \mathcal{A}_{5}$ \\
\hline Liu et al. [19] & Correlation coefficient & -0.4603 & 0.0000 & 0.5198 & 0.1143 & -0.6336 & $\mathcal{A}_{3} \succ \mathcal{A}_{4} \succ \mathcal{A}_{2} \succ \mathcal{A}_{1} \succ \mathcal{A}_{5}$ \\
\hline Luo and Ren [21] & Similarity measure & 0.8221 & 0.8527 & 0.8827 & 0.8492 & 0.7562 & $\mathcal{A}_{3} \succ \mathcal{A}_{2} \succ \mathcal{A}_{4} \succ \mathcal{A}_{1} \succ \mathcal{A}_{5}$ \\
\hline $\begin{array}{l}\text { Maheshwari and } \\
\text { Srivastava [33] }\end{array}$ & Divergence measure & 0.0534 & 0.0454 & 0.0249 & 0.0655 & 0.0621 & $\mathcal{A}_{3} \succ \mathcal{A}_{2} \succ \mathcal{A}_{1} \succ \mathcal{A}_{5} \succ \mathcal{A}_{4}$ \\
\hline Ohlan $[30]$ & Divergence measure & 0.2254 & 0.1395 & 0.0731 & 0.0957 & 0.3216 & $\mathcal{A}_{3} \succ \mathcal{A}_{4} \succ \mathcal{A}_{2} \succ \mathcal{A}_{1} \succ \mathcal{A}_{5}$ \\
\hline $\begin{array}{l}\text { Srivastava and } \\
\text { Maheshwari [34] }\end{array}$ & Divergence measure & 0.1520 & 0.1722 & 0.1125 & 0.1926 & 0.2345 & $\mathcal{A}_{3} \succ \mathcal{A}_{1} \succ \mathcal{A}_{2} \succ \mathcal{A}_{4} \succ \mathcal{A}_{5}$ \\
\hline Garg et al. [31] & Divergence measure & 0.2428 & 0.1922 & 0.1196 & 0.2981 & 0.2685 & $\mathcal{A}_{3} \succ \mathcal{A}_{2} \succ \mathcal{A}_{1} \succ \mathcal{A}_{5} \succ \mathcal{A}_{4}$ \\
\hline Shen et al. [35] & Distance measure & 0.1486 & 0.2104 & 0.1078 & 0.1759 & 0.2969 & $\mathcal{A}_{3} \succ \mathcal{A}_{1} \succ \mathcal{A}_{4} \succ \mathcal{A}_{2} \succ \mathcal{A}_{5}$ \\
\hline Ye $[36]$ & Similarity measure & 0.9084 & 0.9525 & 0.9767 & 0.9360 & 0.9099 & $\mathcal{A}_{3} \succ \mathcal{A}_{2} \succ \mathcal{A}_{4} \succ \mathcal{A}_{5} \succ \mathcal{A}_{1}$ \\
\hline Song et al. [37] & Similarity measure & 0.9492 & 0.9590 & 0.9812 & 0.9522 & 0.9346 & $\mathcal{A}_{3} \succ \mathcal{A}_{2} \succ \mathcal{A}_{4} \succ \mathcal{A}_{1} \succ \mathcal{A}_{5}$ \\
\hline
\end{tabular}

regions based on facilities and a "reference set" $\mathcal{B}$ is designed in terms of CIFS as follows:

$$
B=\left\{\begin{array}{l}
\left(\mathcal{C}_{1},(0.7,0.5),(0.1,0.3)\right), \\
\left(\mathcal{C}_{2},(0.4,0.6),(0.5,0.2)\right), \\
\left(\mathcal{C}_{3},(0.5,0.5),(0.3,0.1)\right), \\
\left(\mathcal{C}_{4},(0.8,0.7),(0.2,0.1)\right)
\end{array}\right\}
$$

During a visit to each region, a team of experts has investigated them and summarized their information in Table 5. The ranking results corresponding to this problem are listed in Table 6 along with several existing MCDM methods [42,44,45,52]. From this table, it is seen that the best alternative remains $\mathcal{A}_{3}$, but the alternative $\mathcal{A}_{1}$ is preferable over $\mathcal{A}_{4}$. In the approaches [17-19,21,30,31,33-37] under IFS environment, it is observed that $\mathcal{A}_{4}$ is preferable over $\mathcal{A}_{1}$. This change in ordering results from the change in the considered environment. Further, the scope of studies under the IFS is more limited than that of CIFS studies.

Example 5.2 [45]. Consider a medical diagnosis prob- 
Table 7. Input data for Example 5.2.

\begin{tabular}{ccccc}
\hline & $\boldsymbol{s}_{\boldsymbol{1}}$ & $\boldsymbol{s}_{\mathbf{2}}$ & $\boldsymbol{s}_{\mathbf{3}}$ & $\boldsymbol{s}_{\mathbf{4}}$ \\
\hline $\mathcal{Q}_{\mathbf{1}}$ & $((0.8,0.7),(0.1,0.2))$ & $((0.9,0.6),(0.1,0.2))$ & $((0.7,0.8),(0.2,0.1))$ & $((0.8,0.7),(0.2,0.1))$ \\
$\mathcal{Q}_{\mathbf{2}}$ & $((0.6,0.4),(0.1,0.5))$ & $((0.4,0.9),(0.5,0.1))$ & $((0.5,0.5),(0.3,0.3))$ & $((0.4,0.9),(0.5,0.1))$ \\
$\mathcal{Q}_{\mathbf{3}}$ & $((0.3,0.8),(0.3,0.1))$ & $((0.8,0.3),(0.1,0.6))$ & $((0.7,0.6),(0.2,0.2))$ & $((0.2,0.7),(0.8,0.2))$ \\
$\mathcal{Q}_{4}$ & $((0.5,0.3),(0.4,0.6))$ & $((0.3,0.1),(0.6,0.3))$ & $((0.8,0.3),(0.1,0.5))$ & $((0.1,0.3),(0.6,0.5))$ \\
\hline
\end{tabular}

Table 8. Comparative analysis of Example 5.2 with existing studies.

\begin{tabular}{|c|c|c|c|c|c|c|}
\hline \multicolumn{7}{|c|}{ Study under CIFS environment } \\
\hline \multicolumn{2}{|c|}{ Approach } & \multicolumn{4}{|c|}{ Measurement value of $\mathcal{P}$ from } & \multirow{2}{*}{ Ranking order } \\
\hline Ref. & Method based on & $\mathcal{Q}_{1}$ & $\mathcal{Q}_{2}$ & $\mathcal{Q}_{3}$ & $\mathcal{Q}_{4}$ & \\
\hline Alkouri and Salleh [42] & Distance measure & 0.0967 & 0.2717 & 0.2867 & 0.3550 & $\mathcal{Q}_{1} \succ \mathcal{Q}_{2} \succ \mathcal{Q}_{3} \succ \mathcal{Q}_{4}$ \\
\hline Rani and Garg [44] & Euclidean distance measure & 0.1194 & 0.2291 & 0.2669 & 0.3004 & $\mathcal{Q}_{1} \succ \mathcal{Q}_{2} \succ \mathcal{Q}_{3} \succ \mathcal{Q}_{4}$ \\
\hline Garg and Rani [45] & Correlation measure & 0.9696 & 0.8486 & 0.8008 & 0.6980 & $\mathcal{Q}_{1} \succ \mathcal{Q}_{2} \succ \mathcal{Q}_{3} \succ \mathcal{Q}_{4}$ \\
\hline Garg and Rani [52] & Similarity measure & 0.8896 & 0.5723 & 0.6037 & 0.4287 & $\mathcal{Q}_{1} \succ \mathcal{Q}_{3} \succ \mathcal{Q}_{2} \succ \mathcal{Q}_{4}$ \\
\hline \multirow[t]{2}{*}{ Proposed method } & Divergence measure & 0.0121 & 0.0608 & 0.0804 & 0.1077 & $\mathcal{Q}_{1} \succ \mathcal{Q}_{2} \succ \mathcal{Q}_{3} \succ \mathcal{Q}_{4}$ \\
\hline & & \multicolumn{4}{|c|}{ Study under IFS environment } & \\
\hline \multicolumn{2}{|c|}{ Approach } & \multicolumn{4}{|c|}{ Measurement value of $\mathcal{P}$ from } & \multirow{2}{*}{ Ranking order } \\
\hline Ref. & Method based on & $\mathcal{Q}_{1}$ & $\mathcal{Q}_{2}$ & $\mathcal{Q}_{3}$ & $\mathcal{Q}_{4}$ & \\
\hline Zeng and Li [17] & Correlation coefficient & 0.9856 & 0.8461 & 0.7959 & 0.7258 & $\mathcal{Q}_{1} \succ \mathcal{Q}_{2} \succ \mathcal{Q}_{3} \succ \mathcal{Q}_{4}$ \\
\hline Ye $[18]$ & Correlation coefficient & 0.9912 & 0.8585 & 0.7265 & 0.6645 & $\mathcal{Q}_{1} \succ \mathcal{Q}_{2} \succ \mathcal{Q}_{3} \succ \mathcal{Q}_{4}$ \\
\hline Liu et al. [19] & Correlation coefficient & 0.8485 & 0.1907 & 0.6608 & -0.0690 & $\mathcal{Q}_{1} \succ \mathcal{Q}_{3} \succ \mathcal{Q}_{2} \succ \mathcal{Q}_{4}$ \\
\hline Luo and Ren [21] & Similarity measure & 0.9642 & 0.7394 & 0.7725 & 0.6538 & $\mathcal{Q}_{1} \succ \mathcal{Q}_{3} \succ \mathcal{Q}_{2} \succ \mathcal{Q}_{4}$ \\
\hline $\begin{array}{l}\text { Maheshwari and } \\
\text { Srivastava [33] }\end{array}$ & Divergence measure & 0.0195 & 0.0523 & 0.0820 & 0.0887 & $\mathcal{Q}_{1} \succ \mathcal{Q}_{2} \succ \mathcal{Q}_{3} \succ \mathcal{Q}_{4}$ \\
\hline Ohlan [30] & Divergence measure & 0.0100 & 0.3090 & 0.3946 & 0.6407 & $\mathcal{Q}_{1} \succ \mathcal{Q}_{2} \succ \mathcal{Q}_{3} \succ \mathcal{Q}_{4}$ \\
\hline $\begin{array}{l}\text { Srivastava and } \\
\text { Maheshwari [34] }\end{array}$ & Divergence measure & 0.0365 & 0.2345 & 0.2345 & 0.2996 & $\mathcal{Q}_{1} \succ \mathcal{Q}_{2}=\mathcal{Q}_{3} \succ \mathcal{Q}_{4}$ \\
\hline Garg et al. [31] & Divergence measure & 0.1024 & 0.2058 & 0.3318 & 0.3170 & $\mathcal{Q}_{1} \succ \mathcal{Q}_{2} \succ \mathcal{Q}_{4} \succ \mathcal{Q}_{3}$ \\
\hline Shen et al. [35] & Distance measure & 0.0308 & 0.2428 & 0.3339 & 0.4386 & $\mathcal{Q}_{1} \succ \mathcal{Q}_{2} \succ \mathcal{Q}_{3} \succ \mathcal{Q}_{4}$ \\
\hline Ye $[36]$ & Similarity measure & 0.9804 & 0.9023 & 0.8000 & 0.8311 & $\mathcal{Q}_{1} \succ \mathcal{Q}_{2} \succ \mathcal{Q}_{4} \succ \mathcal{Q}_{3}$ \\
\hline Song et al. [37] & Similarity measure & 0.9825 & 0.9468 & 0.8931 & 0.8861 & $\mathcal{Q}_{1} \succ \mathcal{Q}_{2} \succ \mathcal{Q}_{3} \succ \mathcal{Q}_{4}$ \\
\hline
\end{tabular}

lem with four diseases $\mathcal{Q}_{1}$ (viral fever), $\mathcal{Q}_{2}$ (malaria), $\mathcal{Q}_{3}$ (typhoid), $\mathcal{Q}_{4}$ (stomach problem) and four symptoms $s_{1}$ (temperature), $s_{2}$ (headache), $s_{3}$ (stomach pain), $s_{4}$ (cough). The rating values of each disease under symptoms are given in Table 7 . The weight of each symptom is taken as $\kappa=(0.30,0.20,0.10,0.40)^{T}$. Consider a patient $\mathcal{P}$ approach to the expert regarding their medical diagnosis. An expert treated this patient as a reference set and rated the values of each symptom in terms of CIFSs, as summarized below:

$$
\mathcal{P}=\left\{\begin{array}{l}
\left(s_{1},(0.8,0.6),(0.1,0.2)\right), \\
\left(s_{2},(0.9,0.7),(0.1,0.2)\right), \\
\left(s_{3},(0.7,0.8),(0.2,0.1)\right), \\
\left(s_{4},(0.6,0.5),(0.2,0.4)\right)
\end{array}\right\} .
$$

The aim here is to diagnose the patient $\mathcal{P}$ and pinpoint the disease. To do so, a number of methods as well as the existing approaches employed [17-19,21,30,31,33$37,42,44,45,52]$ and their results are given in Table 8 . Accordingly, we found that $\mathcal{Q}_{1}$ was infested with diseases as was found by all the methods, demonstrating the feasibility of the approach.

\subsection{Characteristic comparison}

To study the characteristics of the developed method over the existing approaches $[9,11,13,17,30-$ $37,44,45,49]$, we analyzed their characteristics in Table 9. In this table, ' $\checkmark$ ' implies that the corresponding DMP satisfies the criteria such as an ideal alternative required to evaluate the process, measure the degree of discrimination and ability to handle the wider information, etc., whereas ' $x$ ' means that the corresponding method fails. Further, from this table, 
Table 9. The characteristic comparison of different approaches.

\begin{tabular}{|c|c|c|c|c|c|}
\hline Method & $\begin{array}{l}\text { Needs an } \\
\text { ideal } \\
\text { alternative } \\
\text { to compute } \\
\text { the process }\end{array}$ & $\begin{array}{c}\text { Measure the } \\
\text { degree of } \\
\text { discrimination } \\
\text { between } \\
\text { the sets }\end{array}$ & $\begin{array}{l}\text { No unknown } \\
\text { parameter to } \\
\text { choose during } \\
\text { evaluation }\end{array}$ & $\begin{array}{c}\text { Ability to } \\
\text { capture } \\
\text { information } \\
\text { using } \\
\text { complex } \\
\text { numbers }\end{array}$ & $\begin{array}{c}\text { Ability to } \\
\text { handle } \\
\text { two-dimensional } \\
\text { information }\end{array}$ \\
\hline Garg [9] & $\times$ & $\times$ & $\times$ & $\times$ & $\times$ \\
\hline Huang [11] & $x$ & $x$ & $x$ & $x$ & $x$ \\
\hline Chen and Chang [13] & $x$ & $\times$ & $x$ & $\times$ & $x$ \\
\hline Mishra et al. [32] & $\checkmark$ & $\checkmark$ & $x$ & $x$ & $x$ \\
\hline Garg et al. [31] & $\checkmark$ & $\checkmark$ & $x$ & $x$ & $x$ \\
\hline Maheshwari and Srivastava [33] & $\checkmark$ & $\checkmark$ & $x$ & $\times$ & $\times$ \\
\hline Ohlan [30] & $\checkmark$ & $\checkmark$ & $x$ & $\times$ & $x$ \\
\hline Srivastava and Maheshwari [34] & $\checkmark$ & $\checkmark$ & $\times$ & $\times$ & $x$ \\
\hline Shen et al. [35] & $\checkmark$ & $\times$ & $x$ & $x$ & $\times$ \\
\hline $\mathrm{Ye}[36]$ & $\checkmark$ & $\times$ & $x$ & $x$ & $x$ \\
\hline Song et al. [37] & $\checkmark$ & $x$ & $x$ & $x$ & $x$ \\
\hline Zeng and Li [17] & $\checkmark$ & $x$ & $x$ & $x$ & $x$ \\
\hline Rani and Garg [44] & $\checkmark$ & $x$ & $\checkmark$ & $\checkmark$ & $\checkmark$ \\
\hline Garg and Rani [45] & $\checkmark$ & $\times$ & $\checkmark$ & $\checkmark$ & $\checkmark$ \\
\hline Rani and Garg [49] & $\times$ & $\times$ & $\checkmark$ & $\checkmark$ & $\checkmark$ \\
\hline Proposed method & $\checkmark$ & $\checkmark$ & $\checkmark$ & $\checkmark$ & $\checkmark$ \\
\hline
\end{tabular}

it is clearly seen that the methods presented in [30-34] under the IFS environment fail to deal with time periodicity problems. Also, the methods presented in $[17,35-37,44,45,49]$ do not measure the degree of discrimination between the two sets. Further, the DM approaches proposed in [30-34] fail to model complex problems, whereas the range of MDs and NMDs is a unit disc on the complex plane in the presented method. The extension of the above ranges enables the proposed approach also to deal with one-dimensional problems described in [30-34]. Therefore, the developed MCDM approach is more generalized.

\section{Conclusion}

This paper attempted to give an exponential divergence measure for Complex IFSs (CIFSs) to measure the degree of discrimination between two or more CIFSs. Intuitionistic Fuzzy Set (IFS) is generally used by researchers to handle the data. However, CIFS is a more extensive and manageable way to express information and it can represent a wide range of fuzzy information. In the presented work, the range of Membership Degrees (MDs) and Non-Membership Degree (NMDs) was extended from real numbers to complex ones with a unit disc. Accordingly, divergence measures were developed and their relevant properties were studied. It was found that when additional components, i.e., phase terms, were set to zero, then the CIFS theory reduces to IFS theory and hence, the existing approaches under IFS environment are special cases of CIFS study. Further, based on the measure, a Decision-Making (DM) approach was presented to solve the MultiCriteria Decision-Making (MCDM) problems and some practical examples were considered to verify their feasibility in comparison to several existing approaches. The alignment of the proposed approach to the existing studies was demonstrated and its advantages were outlined eliciting the supreme nature of the proposed theory over the existing ones. Based on its advantages, it is concluded that the presented theory can model the uncertainties with more enhancements than those in primitive environments. In the future, we shall extend the application of the proposed measure to diverse fuzzy environments such as Pythagorean set [54-56] and linguistic environment [57-59] as well as different fields of application such as supply chain management, emerging decision problems, risk evaluation, etc. [60-63].

\section{Abbreviation}

IVFS Interval-Valued Fuzzy Set 
IFS Intuitionistic Fuzzy Set

IVIFS Interval-Valued Intuitionistic Fuzzy Set

CFS Complex Fuzzy Set

IVCFS Interval-Valued Complex Fuzzy Sets

CIFS Complex Intuitionistic Fuzzy Set

\section{References}

1. Zadeh, L.A. "Fuzzy sets", Information and Control, 8, pp. 338-353 (1965).

2. Atanassov, K.T. "Intuitionistic fuzzy sets", Fuzzy Sets and Systems, 20, pp. 87-96 (1986).

3. Garg, H. and Kumar, K. "Linguistic interval-valued atanassov intuitionistic fuzzy sets and their applications to group decision-making problems", IEEE Transactions on Fuzzy Systems, 27(12), pp. 2302-2311 (2019). DOI: 10.1109/TFUZZ.2019.2897961

4. Ramot, D., Milo, R., Fiedman, M., and Kandel, A. "Complex fuzzy sets", IEEE Transactions on Fuzzy Systems, 10(2), pp. 171-186 (2002).

5. Alkouri, A. and Salleh, A. "Complex intuitionistic fuzzy sets", 2nd International Conference on Fundamental and Applied Sciences, 1482, pp. 464-470 (2012).

6. Garg, H. and Rani, D. "Complex interval- valued intuitionistic fuzzy sets and their aggregation operators", Fundamenta Informaticae, 164(1), pp. 61-101 (2019).

7. Xu, Z.S. and Yager, R.R. "Some geometric aggregation operators based on intuitionistic fuzzy sets", International Journal of General Systems, 35, pp. 417-433 (2006).

8. Xu, Z.S. "Intuitionistic fuzzy aggregation operators", IEEE Transactions on Fuzzy Systems, 15, pp. 11791187 (2007).

9. Garg, H. "Generalized intuitionistic fuzzy interactive geometric interaction operators using Einstein t-norm and t-conorm and their application to decision making", Computers and Industrial Engineering, 101, pp. 53-69 (2016).

10. Garg, H. and Singh, S. "A novel triangular interval type-2 intuitionistic fuzzy sets and their aggregation operators", Iranian Journal of Fuzzy Systems, 15(5), pp. 69-93 (2018).

11. Huang, J.Y. "Intuitionistic fuzzy Hamacher aggregation operator and their application to multiple attribute decision making", Journal of Intelligent and Fuzzy Systems, 27, pp. 505-513 (2014).

12. Jasemi, M. and Ahmadi, E. "A new fuzzy ELECTRE based multiple criteria method for personnel selection", Scientia Iranica, E, 25(2), pp. 943-953 (2018).

13. Chen, S.M. and Chang, C.H. "Fuzzy multiattribute decision making based on transformation techniques of intuitionistic fuzzy values and intuitionistic fuzzy geometric averaging operators", Information Sciences, 352-353, pp. 133-149 (2016).
14. Arora, R. and Garg, H. "Prioritized averaging/geometric aggregation operators under the intuitionistic fuzzy soft set environment", Scientia Iranica, 25(1), pp. 466-482 (2018).

15. Wan, S. and Xu, J. "A method for multi-attribute group decision making with triangular intuitionistic fuzzy numbers and application to trustworthy service selection", Scientia Iranica, E, 24(2), pp. 794-807 (2017).

16. Tang, J. and Meng, F. “An approach to interval-valued intuitionistic fuzzy decision making based on induced generalized symmetrical Choquet Shapley operator", Scientia Iranica, D, 25(3), pp. 1456-1470 (2018).

17. Zeng, W. and Li, H. "Correlation coefficient of intuitionistic fuzzy sets", Journal of Industrial Engineering International, 3(5), pp. 33-40 (2007).

18. Ye, J. "Cosine similarity measures for intuitionistic fuzzy sets and their applications", Mathematical and Computer Modelling, 53(1-2), pp. 91-97 (2011).

19. Liu, B., Shen, Y., Mu, L., Chen, X. and Chen, L. "A new correlation measure of the intuitionistic fuzzy sets", Journal of Intelligent \& Fuzzy Systems, 30, pp. 1019-1028 (2016).

20. Kumar, K. and Garg, H. "TOPSIS method based on the connection number of set pair analysis under interval-valued intuitionistic fuzzy set environment", Computational and Applied Mathematics, 37(2), pp. 1319-1329 (2018).

21. Luo, L. and Ren, H. "A new similarity measure of intuitionistic fuzzy set and application in MADM problem", AMSE Series Advances A, 59, pp. 204-223 (2016).

22. Garg, H. and Kumar, K. "A novel exponential distance and its based TOPSIS method for interval-valued intuitionistic fuzzy sets using connection number of SPA theory", Artificial Intelligence Review, pp. 1-30 (2018). DOI: 10.1007/s10462-018-9668-5

23. Garg, H. "Novel intuitionistic fuzzy decision making method based on an improved operation laws and its application", Engineering Applications of Artificial Intelligence, 60, pp. 164-174 (2017).

24. Garg, H. "Intuitionistic fuzzy hamacher aggregation operators with entropy weight and their applications to multi-criteria decision-making problems", Iranian Journal of Science and Technology, Transactions of Electrical Engineering, 43(3), pp. 597-613 (2019).

25. Kullback, S. and Leibler, R.A. "On information and sufficiency", Annals of Mathematical Statistics, 22, pp. 79-86 (1951).

26. Bhandari, D. and Pal, N.R. "Some new information measures for fuzzy sets", Information Sciences, 67, pp. 209-228 (1993). 
27. Vlachos, I.K. and Sergiadis, G.D. "Intuitionistic fuzzy information - application to pattern recognition", Pattern Recognition Letters, 28(2), pp. 197-206 (2007).

28. Garg, H. "An improved cosine similarity measure for intuitionistic fuzzy sets and their applications to decision-making process", Hacettepe Journal of Mathematics and Statistics, 47(6), pp. 1585-1601 (2018).

29. Garg, H. and Kumar, K. "An advanced study on the similarity measures of intuitionistic fuzzy sets based on the set pair analysis theory and their application in decision making", Soft Computing, 22(15), pp. 49594970 (2018).

30. Ohlan, A. "Intuitionistic fuzzy exponential divergence: Application in multi-attribute decision making", Journal of Intelligent \& Fuzzy Systems, 30, pp. 1519-1530 (2016).

31. Garg, H., Agarwal, N., and Tripathi, A. "A novel generalized parametric directed divergence measure of intuitionistic fuzzy sets with its application", Annals of Fuzzy Mathematics and Informatics, 13(6), pp. 703727 (2017)

32. Mishra, A.R., Kumari, R., and Sharma, D.K. "Intuitionistic fuzzy divergence measure-based multicriteria decision-making method", Neural Computing \& Applications, pp.1-16 (2017). DOI: 10.1007/s00521017-3187-1

33. Maheshwari, S. and Srivastava, A. "Study on divergence measures for intuitionistic fuzzy sets and its application in medical diagnosis", Journal of Applied Analysis and Computation, 6(3), pp. 772-789 (2016).

34. Srivastava, A. and Maheshwari, S. "Decision making in medical investigations using new divergence measures for intuitionistic fuzzy sets", Iranian Journal of Fuzzy Systems, 13(1), pp. 25-44 (2016).

35. Shen, F., Ma, X., Li, Z., Xu, Z.S., and Cai, D. "An extended intuitionistic fuzzy TOPSIS method based on a new distance measure with an application to credit risk evaluation", Information Sciences, 428, pp. 105119 (2018).

36. Ye, J. "Similarity measures of intuitionistic fuzzy sets based on cosine function for the decision making of mechanical design schemes", Journal of Intelligent \& Fuzzy Systems, 30, pp. 151-158 (2016).

37. Song, Y., Wang, X., Quan, W., and Huang, W. "A new approach to construct similarity measure for intuitionistic fuzzy sets", Soft Computing, 23(6), pp. 1985

38. Ramot, D., Friedman, M., Langholz, G., and Kandel, A. "Complex fuzzy logic", IEEE Transactions on Fuzzy Systems, 11(4), pp. 450-461 (2003).

39. Dick, S., Yager, R.R. and Yazdanbakhsh, O. "On Pythagorean and complex fuzzy set operations", IEEE Transactions on Fuzzy Systems, 24(5), pp. 1009-1021 (2016).
40. Yager, R.R. and Abbasov, A.M. "Pythagorean membeship grades, complex numbers and decision making", International Journal of Intelligent Systems, 28, pp. 436-452 (2013).

41. Yazdanbakhsh, O. and Dick, S. "A systematic review of complex fuzzy sets and logic", Fuzzy Sets and Systems, 338, pp. 1-22 (2018).

42. Alkouri, A.U.M. and Salleh, A.R. "Complex Atanassov's intuitionistic fuzzy relation", Abstract and Applied Analysis, 2013, Article ID 287382, 18 pages (2013).

43. Kumar, T. and Bajaj, R.K. "On complex intuitionistic fuzzy soft sets with distance measures and entropies", Journal of Mathematics, 2014, Article ID 972198, 12 pages (2014).

44. Rani, D. and Garg, H. "Distance measures between the complex intuitionistic fuzzy sets and its applications to the decision - making process", International Journal for Uncertainty Quantification, 7(5), pp. 423439 (2017).

45. Garg, H. and Rani, D. "A robust correlation coefficient measure of complex intuitionistic fuzzy sets and their applications in decision-making", Applied Intelligence, 49(2), pp. 496-512 (2019).

46. Garg, H. and Rani, D. "Some generalized complex intuitionistic fuzzy aggregation operators and their application to multicriteria decision-making process", Arabian Journal for Science and Engineering, 44(3), pp. 2679-2698 (2019).

47. Garg, H. and Rani, D. "Robust averaging-geometric aggregation operators for complex intuitionistic fuzzy sets and their applications to MCDM process", Arabian Journal for Science and Engineering, 45(3), pp. 2017-2033 (2020). DOI: 10.1007/s13369-019-03925-4

48. Quran, A.A. and Hassan, N. "The complex neutrosophic soft expert set and its application in decision making", Journal of Intelligent \& Fuzzy Systems, 34, pp. 569-582 (2018).

49. Rani, D. and Garg, H. "Complex intuitionistic fuzzy power aggregation operators and their applications in multi-criteria decision-making", Expert Systems, 35(6), p. e12325 (2018).

50. Garg, H. and Rani, D. "New generalized Bonferroni mean aggregation operators of complex intuitionistic fuzzy information based on Archimedean t-norm and t-conorm", Journal of Experimental and Theoretical Artificial Intelligence, 32(1), pp. 81-109 (2020). DOI: 10.1080/0952813X.2019.1620871

51. Garg, H. and Rani, D. "Exponential, logarithmic and compensative generalized aggregation operators under complex intuitionistic fuzzy environment", Group Decision and Negotiation, 28(5), pp. 991-1050 (2019).

52. Garg, H. and Rani, D. "Some results on information measures for complex intuitionistic fuzzy sets", International Journal of Intelligent Systems, 34(10), pp. 2319-2363 (2019). 
53. Yingming, W. "Using the method of maximizing deviations to make decision for multi-indices", Journal of Systems Engineering and Electronics, 8(3), pp. 21-26 (1997).

54. Wang, J., Gao, H., and Wei, G.W. "The generalized Dice similarity measures for Pythagorean fuzzy multiple attribute group decision making", International Journal of Intelligent Systems, 34(6), pp. 1158-1183 (2019).

55. Tang, X.Y. and Wei, G.W. "Multiple attribute decision-making with Dual hesitant Pythagorean fuzzy information", Cognitive Computation, 11(2), pp. 193211 (2019).

56. Tang, M., Wang, J., Lu, J.P., Wei, G.W., Wei, C., and Wei, Y. "Dual hesitant Pythagorean fuzzy heronian mean operators in multiple attribute decision making", Mathematics, 7(4), pp. 344 (2019).

57. Arora, R. and Garg, H. "Group decision-making method based on prioritized linguistic intuitionistic fuzzy aggregation operators and its fundamental properties", Computational and Applied Mathematics, 38(2), pp. 1-36 (2019).

58. Garg, H. and Nancy, "Linguistic single-valued neutrosophic power aggregation operators and their applications to group decision-making problems", IEEE/CAA Journal of Automatic Sinica, 7(2), pp. 546-558 (2020). DOI: $10.1109 /$ JAS.2019.1911522

59. Garg, H. and Kumar, K. "Multiattribute decision making based on power operators for linguistic intuitionistic fuzzy set using set pair analysis", Expert Systems, 36(4), p. e12428 (2019). DOI: 10.1111/exsy. 12428

60. Tang, X.Y., Wei, G.W., and Gao, H. "Models for multiple attribute decision making with interval-valued Pythagorean fuzzy Muirhead mean operators and their application to green suppliers selection", Informatica, 30(1), pp. 153-186 (2019).

61. Peng, X., Dai, J., and Garg, H. "Exponential operation and aggregation operator for q-rung orthopair fuzzy set and their decision-making method with a new score function", International Journal of Intelligent Systems, 33(11), pp. 2255-2282 (2018).

62. Peng, X.D. and Garg, H. "Algorithms for intervalvalued fuzzy soft sets in emergency decision making based on WDBA and CODAS with new information measure", Computers \& Industrial Engineering, 119, pp. 439-452 (2018).

63. Garai, T. and Garg, H. "Multi-objective linear fractional inventory model with possibility and necessity constraints under generalized intuitionistic fuzzy set environment", CAAI Transactions on Intelligence Technology, 4(3), pp. 175-181 (2019).

\section{Biographies}

Harish Garg is working as an Associate Professor with School of Mathematics at Thapar Institute of Engineering \& Technology (Deemed University) Patiala, Punjab, India. He has supervised $07 \mathrm{PhD}$ dissertations and has authored over 320 papers with SCI published in refereed International Journals including Information Sciences, IEEE Transactions on Fuzzy Systems, International Journal of Intelligent Systems, IEEE/CAA Journal of Automatic Sinica, IEEE Transactions On Emerging Topics In Computational Intelligence, Cognitive Computation, Artificial Intelligence Review, Applied Soft Computing, Experts Systems with Applications, Journal of Manufacturing Systems, Applied Mathematics \& Computations, ISA Transactions, Applied Intelligence, Computer and Industrial Engineering, Soft Computing, Computer and Operations Research, Journal of Experimental \& Theoretical Artificial Intelligence, International Journal of Uncertainty, Fuzziness and Knowledge-based Systems, Journal of Industrial and Management Optimization, International Journal of Uncertainty Quantification, and many more. He is the recipient of the TopCited Paper by India-based Author (2015-2019) from Elsevier Publisher. Dr. Garg is the Editor-in-Chief of Annals of Optimization Theory \& Practice, Journal of Computational and Cognitive Engineering. He is also Associate Editors for several reputed journal such as Soft Computing, Journal of Intelligent \& Fuzzy Systems, International Journal of Computational Intelligence Systems, Kybernetes, Technological and Economic Development of Economy, Mathematical Problems in Engineering, Journal of Industrial \& Management Optimization, Complexity, Complex and Intelligent Systems, CAAI Transactions on Intelligence Technology and so on. His Google citations are over 12000 with h-index of 63 . His research interests are in the fields of computational Iitelligence, reliability analysis, multi-criteria decision making, fuzzy decision making, Pythagorean fuzzy sets, computing with words and soft Computing.

Dimple Rani received her doctoral degree from Thapar Institute of Engineering \& Technology (Deemed University) Patiala, Punjab, India under the supervision of Dr. Harish Garg. She obtained her Master degree in Mathematics during 2013-2015 from Khalsa College Patiala, Punjab, India. Currently, her research interests are uncertainty, complex systems, and decision-making theory. She has published 20 article papers in different top-reputed SCI journals. 